\title{
ANALYTICAL VALIDATION OF THE YOUNG-DUPRÉ LAW FOR EPITAXIALLY-STRAINED THIN FILMS
}

\author{
ELISA DAVOLI AND PAOLO PIOVANO
}

\begin{abstract}
We present here an analysis of the regularity of minimizers of a variational model for epitaxially strained thin-films identified by the authors in the companion paper [7]. The regularity of energetically-optimal film profiles is studied by extending previous methods and by developing new ideas based on transmission problems. The achieved regularity results relate to both the Stranski-Krastanow and the Volmer-Weber modes, the possibility of different elastic properties between the film and the substrate, and the presence of the surface tensions of all three involved interfaces: film/gas, substrate/gas, and film/substrate. Finally, geometrical conditions are provided for the optimal wetting angle, i.e., the angle formed at the contact point of films with the substrate. In particular, the Young-Dupré law is shown to hold, yielding what appears to be the first analytical validation of such law for a thin-film model in the context of Continuum Mechanics.
\end{abstract}

\section{Contents}

1. Introduction 1

1.1. The Young-Dupré law in Fluid Mechanics . . . . . . . . . . . . . . 2

1.2. The thin-film model . . . . . . . . . . . . . . . . 3

1.3. Wettability and growth modes . . . . . . . . . . . . 5 5

1.4. Organization of the paper and methodology . . . . . . . . . 5 5

2. Main results $\quad 7$

2.1. Mathematical setting ............... . . 7

2.2. Statement of the main results . . . . . . . . . . . . . . 10

3. Properties of local minimizers 14

3.1. Internal-ball condition . . . . . . . . . . . . . . . . . . . 14

3.2. Decay estimate . . . . . . . . . . . . . . 15

4. Contact-Angle conditions 21

Island borders . . . . . . . . . . . . . . . . . . . . . 25

Valleys with no vanishing contact angles . . . . . . . . . . . 28

2010 Mathematics Subject Classification. 35J50, 49J10, 74K35.

Key words and phrases. Young-Dupré, contact angle, wetting, triple junctions, thin films, sharpinterface model, transmission problems, $\Gamma$-convergence. 
Valleys with one vanishing contact angle . . . . . . . . . . . . 29

Jumps: Island borders . . . . . . . . . . . . . . . . . . . . . 30

Jumps: Valleys with no vanishing contact angles . . . . . . . . . . . . 32

Jumps: Valleys with one vanishing contact angle . . . . . . . . . . . . 32

5. Regularity of local minimizers 34

Acknowledgements $\quad 35$

References $\quad 35$

\section{INTRODUCTION}

Originally formulated in the context of Fluid Mechanics and sessile liquid drops [10, 35], the Young-Dupré law characterizes the contact angle formed by drops at any touching point with their supporting surfaces (see Figure 1). As this condition involves both the tension of supporting surfaces and the contact angles of drops (see Subsection 1.1), the law is often used to determine the unknown surface tension of certain materials by measuring the contact angles formed by different probe liquids.

The use of this law is however not only restricted to liquid drops, but it has been naturally extended to epitaxy, i.e., to the deposition of crystalline films on crystalline substrates [27, Section 4.2.2]. Contact-angle conditions are in fact often essential for studying multiple-phase systems, as they represent the crucial boundary conditions for characterizing interface morphologies at (triple) junctions [2, 33]. A crucial difference between the setting of sessile drops and the one of thin-film deposition, though, is that in the latter elasticity has also to be taken into account as it might strongly affect the profile of the film. Indeed, the mismatch between the crystalline lattices of the film and the substrate can induce large stresses in the film. In order to release such energy the atoms of the film move from their crystalline equilibrium to reach more favorable arrangements [14].

Despite the applications of the Young-Dupré law to elastic solids, a mathematical justification in the context of Continuum Mechanics seems to be missing in the Literature. In this regard we refer the reader to [32] for a discussion on whether the presence of stresses modifies contact angles or not. In this paper we provide such mathematical validation in linear elasticity in the context of thin films starting from the models introduced in [7]. Among our results we in particular find that the classical contact angles determined by the Young-Dupré law are not impacted by the singular elastic fields present at the wedges of the contact corners.

The purpose of this paper is therefore twofold. First, we show that optimal thin-film profiles of the variational heteroepitaxial thin-film model identified in [7] (see (1.2)) satisfy the Young-Dupré law for angles $\theta \in[0, \pi / 2]$ (see Theorem 2.3). Second, in Theorem 2.4 the regularity of the profile of minimizing configurations is assessed.

1.1. The Young-Dupré law in Fluid Mechanics. The first formulation of the law dates back to 1805 and is due to Thomas Young [35] who derived it by computing the 
mechanical equilibrium of drops resting on planar surfaces under the action of the surface tensions $\gamma_{f}, \gamma_{s}, \gamma_{f s}$ of the three involved interfaces, respectively, the drop/gas interface, the substrate/gas interface, and the drop/substrate interface. Notice that we use here the subscript $f$ because in our setting drops coincide with film material. Subsequently in 1869 a zero-angle condition for the case in which $\gamma_{f} \leq \gamma_{s}-\gamma_{f s}$ (also called wetting criterion in [28]) has been included in the relation by Anthanase Dupré and Paul Dupré (see [10]). A formulation of the law that includes both the contributions of [35] and [10] is

$$
\cos \theta=\frac{\min \left\{\gamma_{f}, \gamma_{s}-\gamma_{f s}\right\}}{\gamma_{f}},
$$

where $\theta$ is the contact angle between planar surface of the substrate and the film profile (see Figure 1).

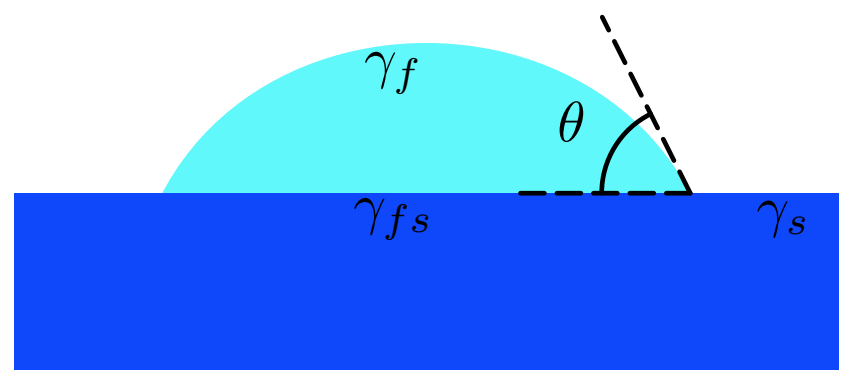

Figure 1. Contact angle of a sessile drop.

In 1877 Carl Friedrich Gauss [17] introduced a free energy consisting of four terms: a free surface energy related to the boundary of the drop detached from the substrate, a wetting energy accounting for the adhesion of the drop to the supporting surface and depending of an adhesion coefficient $\sigma$, a gravitational energy, and a Lagrange multiplier to include a constraint on the volume of the drop. We recall that a law which includes this adhesion coefficient $\sigma$ has been formulated by Pierre Simon Laplace in [24] also starting from the ideas in [35]. This law which is often referred to as Young-Laplace law in the context of capillarity problems, i.e., problems related to fluids in containers, can be stated as $\nu_{D} \cdot \nu_{C}=\sigma$, where $\nu_{D}$ and $\nu_{C}$ are the exterior normal to the drop and the container, respectively. We observe that (1.1) is equivalent to the Young-Laplace law when $-\sigma$ corresponds to the right-hand side of (1.1).

However, also the results in the Literature related to the Young-Laplace law seem not to include elasticity. In particular, in [5] the authors prove that, if $\sigma \in(-1,0)$, than the detached boundary of the minimizing drops of the Gauss free energy is the graph of a function describing the thickness of the drop. This result, although in a different context, is in accordance with our analysis. In fact we assume that the admissible film profiles are graphs of height functions and the conditions that we need to impose on $\gamma_{f}$, $\gamma_{s}, \gamma_{f s}$ are such that the right-hand side of (1.1) belongs to $[0,1]$ (where, though, the boundary values can be included in our analysis). For more general conditions on the adhesion coefficient $\sigma$ we refer the reader to [1] and [8], where every set of finite perimeter is an admissible drop, and the boundary regularity of optimal drops is studied also in the presence of anisotropy. 
1.2. The thin-film model. The first rigorous validation of a thin-film energy as $\Gamma$-limit of the transition-layer model of [29] was performed in the seminal paper [12]. In the companion paper [7] we have provided a variational model taking into account the (possible) different elastic properties of the film and substrate materials, and thus particularly apt to describe heteroepitaxy, i.e., the deposition of a material different from the one of the substrate.

In order to describe the model identified in [7], we need to introduce some notation. As in the seminal paper [29] we model substrate and the film as continua, work in the setting of linear elasticity, and consider two-dimensional profiles (or three-dimensional configurations with planar symmetry). We assume that interface between film and substrate is a subset of the $x$-axis, and we denote by the height function $h:[a, b] \rightarrow[0,+\infty)$ with $b>a>0$ the film thickness. The region occupied by the film and the substrate material is described by the subgraph of $h$, namely by the set

$$
\Omega_{h}:=\{(x, y): a<x<b, y<h(x)\},
$$

whilst the film profile is encoded by the graph

$$
\Gamma_{h}:=\partial \Omega_{h} \cap((a, b) \times \mathbb{R}) .
$$

The material displacement and its associated strain-tensor are denoted by $u: \Omega_{h} \rightarrow \mathbb{R}^{2}$, and its symmetric part of the gradient, namely

$$
E u:=\operatorname{sym} \nabla u,
$$

respectively. As in [12], our description will include non-smooth profiles, and the height function will be assumed to be lower semicontinuous and with bounded pointwise variation. We will adopt the notation

$$
\tilde{\Gamma}_{h}:=\partial \bar{\Omega}_{h} \cap((a, b) \times \mathbb{R}),
$$

and $\Gamma_{h}^{c u t}$ to identify the set of cuts in the profile of $h$, namely $\Gamma_{h}^{c u t}:=\Gamma_{h} \backslash \tilde{\Gamma}_{h}$.

The lattice mismatch between the film and the substrate materials is known to induce large stresses and thus to play a major role in heteroepitaxy [14]. In our model the lattice mismatch is represented by means of a parameter $e_{0} \geq 0$, and by the assumption that the minimum of the energy is attained at

$$
E_{0}(y):= \begin{cases}e_{0}\left(\mathbf{e}_{\mathbf{1}} \odot \mathbf{e}_{\mathbf{1}}\right) & \text { if } y \geq 0 \\ 0 & \text { otherwise }\end{cases}
$$

where $\left(\mathbf{e}_{\mathbf{1}}, \mathbf{e}_{\mathbf{2}}\right)$ is the standard basis of $\mathbb{R}^{2}$. In the following we refer to $E_{0}$ as the mismatch strain.

The model considered in this paper, and rigorously validated in [7], is the energy functional $\mathcal{F}$, defined for any film configuration $(u, h)$ as

$$
\begin{aligned}
\mathcal{F}(u, h)=\int_{\Omega_{h}} W_{0}(y, E u(x, y) & \left.-E_{0}(y)\right) d x d y \\
& +\int_{\tilde{\Gamma}_{h}} \varphi(y) d \mathcal{H}^{1}+\gamma_{\mathrm{fs}}(b-a)+2 \gamma_{\mathrm{f}} \mathcal{H}^{1}\left(\Gamma_{h}^{c u t}\right),
\end{aligned}
$$

where the surface density $\varphi$ is given by

$$
\varphi(y):= \begin{cases}\gamma_{f} & \text { if } y>0 \\ \min \left\{\gamma_{f}, \gamma_{s}-\gamma_{f s}\right\} & \text { otherwise }\end{cases}
$$


with

$$
\gamma_{f}>0, \quad \gamma_{s}>0, \quad \text { and } \quad \gamma_{s}-\gamma_{f s} \geq 0
$$

In the expression above, the elastic energy density $W_{0}: \mathbb{R} \times \mathbb{M}_{\text {sym }}^{2 \times 2} \rightarrow[0,+\infty)$ is defined as

$$
W_{0}(y, E):=\frac{1}{2} E: \mathbb{C}(y) E
$$

for every $(y, E) \in \mathbb{R} \times \mathbb{M}_{\text {sym }}^{2 \times 2}$, where $\mathbb{C}(y)$ is the elasticity tensor, satisfying

$$
\mathbb{C}(y):= \begin{cases}\mathbb{C}_{f} & \text { if } y>0 \\ \mathbb{C}_{s} & \text { otherwise }\end{cases}
$$

and such that

$$
E: \mathbb{C}(y) E>0
$$

for every $y \in \mathbb{R}$ and $E \in \mathbb{M}_{\text {sym }}^{2 \times 2}$. The fourth-order tensors $\mathbb{C}_{f}$ and $\mathbb{C}_{s}$ are symmetric and positive-definite, and we allow them to be possibly different to include the case of a different elastic behavior for the film and the substrate. An alternative formulation of heteroepitaxy is to consider $E_{0} \equiv 0$ in (1.2), and to impose a transmission Dirichlet condition at the interface between film and substrate. We refer to Remark 2.5 to see that the two corresponding minimum problems are equivalent. As already highlighted in [7], energy functionals of the form (1.2) represent the competition between the roughening effect of the elastic energy and the regularizing effect of the surface energy that characterize the formation of such crystal microstructures (see [14, 16, 18] and [11] for the related problem of crystal cavities) and are thus related to the study of Stress-Driven Rearrangement Instabilities (SDRI) [18]. We refer to [7] and the references therein for an overview on the Literature on variational models in epitaxy and on related SDRI models. As already mentioned at the beginning of this subsection a similar functional to (1.2) was derived in [12] by $\Gamma$-convergence from the transition-layer model introduced in [29] in the case in which $\mathbb{C}_{f}=\mathbb{C}_{s}$, and $\gamma_{f s}=0$. We observe here that in [12] the regularity of the local minimizers of such energy is studied for isotropic film and substrate in the case in which $\gamma_{f} \leq \gamma_{s}$, and the local minimizers are shown to be smooth outside of finitely many cusps and cuts and to form zero contact angles with the substrate (see also [4, 11]). We point out that the functional in [12], when restricted to the regime $\gamma_{f} \leq \gamma_{s}$ did not present any discontinuity along the film/substrate interface contained in the $x$-axis. The same applies for the energy in [15]. In our more general setting, instead, (1.2) always presents a sharp discontinuity with respect to the elastic tensors. Additionally the geometrical and regularity results of this paper include the dewetting regime, $\gamma_{f}>\gamma_{s}-\gamma_{f s}$, for which the surface tension is also discontinuous.

1.3. Wettability and growth modes. The importance of determining on which parameters contact angles in epitaxial growth depend, and of precisely characterizing their amplitude, resides on the need in applications to control the film adherence to substrates. The film adherence, that depends on the chemical interactions between the constituents of the two materials, is also referred to as film wettability.

Zero contact angles correspond to complete wetting that occurs when an infinitesimal thin layer of film atoms, the wetting layer, spreads freely on the substrate and covers it. Positive angles instead represent the so called situation of nonspreading films, in which the substrate is partially exposed [36]. Since contact angles represent the degree of the wettability of the film, they are in general also called wetting angles. 
It is exactly because of their various possible morphologies and wettability properties that thin films play nowadays a key role in an ever-growing number of technologies which range from optoelectronics to semiconductor devices, and from solid oxide fuel/hydrolysis cells to photovoltaic devices. In fact, different modes of growth relate to different film wettability: Volmer-Weber (VW) mode, in which separated islands form on top of the substrate, or situations in which the substrate is completely covered such as in the Frankvan der Merwe (FM) and Stranski-Krastanov (SK) modes. FM and SK differ as FM consists in a layer-by-layer growth (next level starting only upon completion of previous layers), while SK presents islands which are nucleated on top of a wetting layer [27].

Therefore, a large effort has been played at the engineering stage to improve the accuracy with which the resulting processed films correspond to the designed geometries. Any advancement in the modeling that improves the engineering of pre-determined profile shapes has therefore a direct economical impact as it contributes to saving computational time needed for simulations, and to reducing the waste of material used in the current work-intensive and expensive trial-and-error production. We notice here that as a byproduct of our analysis, we also deduce that the VW thin-film mode (which corresponds to a positive wetting angle) is exhibited if and only if $\gamma_{f}>\gamma_{s}-\gamma_{f s}$.

1.4. Organization of the paper and methodology. The paper is organized as follows. In Section 2 we introduce the mathematical setting and we rigorously state our main results (see Theorems 2.3, and 2.4).

In Section 3, starting from the preliminary regularity results proved in [7], we develop a novel strategy for deriving contact-angle conditions. The originality of the method consists in implementing in our thin-film setting some ideas used for transmission problems, that rely on a decomposition formula established in [26], as well as on the properties of the Mellin transform and of the operator pencil (see [25]). In particular, by using the results in [22] we prove in Proposition 3.6 a decay estimate for the displacements corresponding to local minimizers of $\mathcal{F}$.

In Section 4, in view of Proposition 3.6 we are able to perform a blow-up argument at the film/substrate contact points and to pass to the limit in the Euler equation satisfied by local minimizers (by considering variations only with respect to the profile functions). Among the contact points $Z_{h}$ of minimal profiles $h$ we distinguish the isolated ones from the extrema of non-degenerate intervals in $Z_{h}$, and we refer to the first as valleys and to the latter as island borders. Careful choices of suitable competitors for the minimal profile functions with respect to the different cases of valleys and island borders allow in Proposition 4.1 to identify corresponding contact-angle conditions. In particular the conditions proved in Proposition 4.1 include the Young-Dupré law for the wetting regime, $\gamma_{f} \leq \gamma_{s}-\gamma_{f s}$. For the dewetting regime, $\gamma_{f}>\gamma_{s}-\gamma_{f s}$, the Young-Dupré law is obtained in Theorem 2.3 by a further comparison argument, that shows that angles smaller than the one characterized in (1.1) are not energetically convenient in this regime. As a byproduct of our results we also obtain that in the dewetting regime there are no valleys, and hence, that islands are separated.

Finally, in Section 5 an adaptation of the proof strategy of Theorem 2.3, together with improved decay estimates along the lines of [12], allow to reach in Theorem 2.4 the final regularity results for local minimizers. 


\section{MAIN RESUlts}

2.1. Mathematical setting. We recall in this subsection the main definitions and the notation used throughout this paper and in [7]. We begin by characterizing the admissible film profiles. The set $A P$ of admissible film profiles in $(a, b)$ is denoted by

$A P(a, b):=\{h:[a, b] \rightarrow[0,+\infty): h$ is lower semicontinuous and $\operatorname{Var} h<+\infty\}$,

where $\operatorname{Var} h$ denotes the pointwise variation of $h$, namely,

$$
\begin{aligned}
& \text { Var } h:=\sup \left\{\sum_{i=1}^{n}\left|h\left(x_{i}\right)-h\left(x_{i-1}\right)\right|\right. \\
& \left.P:=\left\{x_{1}, \ldots, x_{n}\right\} \text { is a partition of }[a, b]\right\} .
\end{aligned}
$$

We recall that for every lower semicontinuous function $h:[a, b] \rightarrow[0,+\infty)$, to have finite pointwise variation is equivalent to the condition

$$
\mathcal{H}^{1}\left(\Gamma_{h}\right)<+\infty
$$

where

$$
\Gamma_{h}:=\partial \Omega_{h} \cap((a, b) \times \mathbb{R}) .
$$

For every $h \in A P(a, b)$, and for every $x \in(a, b)$, consider the left and right limits

$$
h\left(x^{ \pm}\right):=\lim _{z \rightarrow x^{ \pm}} h(z)
$$

we define

and

$$
h^{-}(x):=\min \left\{h\left(x^{+}\right), h\left(x^{-}\right)\right\}=\liminf _{z \rightarrow x} h(z)
$$

$$
h^{+}(x):=\max \left\{h\left(x^{+}\right), h\left(x^{-}\right)\right\}=\limsup _{z \rightarrow x} h(z) .
$$

In the following $\operatorname{Int}(A)$ denotes the interior part of a set $A$. Let us now recall some properties of height functions $h \in A P(a, b)$, regarding their graphs $\Gamma_{h}$, their subgraphs $\Omega_{h}$, the film and the substrate parts of the subgraph,

$$
\Omega_{h}^{+}:=\Omega_{h} \cap\{y>0\}
$$

and

respectively, and the sets

$$
\Omega_{h}^{-}:=\Omega_{h} \cap\{y \leq 0\}
$$

$$
\tilde{\Gamma}_{h}:=\partial \bar{\Omega}_{h} \cap((a, b) \times \mathbb{R}) .
$$

Any $h \in A P(a, b)$ satisfies the following assertions (see [12, Lemma 2.1]):

1. $\Omega_{h}^{+}$has finite perimeter in $((a, b) \times \mathbb{R})$,

2. $\Gamma_{h}=\left\{(x, y): a<x<b, h(x)<y<h^{+}(x)\right\}$,

3. $h^{-}$is lower semicontinuous and $\operatorname{Int}(\bar{\Omega})=\left\{(x, y): a<x<b, y<h^{-}(x)\right\}$,

4. $\tilde{\Gamma}_{h}=\left\{(x, y): a<x<b, h^{-}(x) \leq y \leq h^{+}(x)\right\}$,

5. $\Gamma_{h}$ and $\tilde{\Gamma}_{h}$ are connected.

We now characterize various portions of $\Gamma_{h}$. To this aim we denote the jump set of a function $h \in A P(a, b)$, i.e., the set of its profile discontinuities, by

$$
J(h):=\left\{x \in(a, b): h^{-}(x) \neq h^{+}(x)\right\},
$$

whereas the set identifying vertical cuts in the graph of $h$ is given by

$$
C(h):=\left\{x \in(a, b): h(x)<h^{-}(x)\right\} .
$$


The graph $\Gamma_{h}$ of a height function $h$ is then characterized by the decomposition

$$
\Gamma_{h}=\Gamma_{h}^{j u m p} \sqcup \Gamma_{h}^{c u t} \sqcup \Gamma_{h}^{\text {graph }},
$$

where $\sqcup$ denotes the disjoint union, and

$$
\begin{aligned}
\Gamma_{h}^{\text {jump }} & :=\overline{\left\{(x, y): x \in(a, b) \cap J(h), h^{-}(x) \leq y \leq h^{+}(x)\right\}}, \\
\Gamma_{h}^{\text {cut }} & :=\left\{(x, y): x \in(a, b) \cap C(h), h(x) \leq y<h^{-}(x)\right\}, \\
\Gamma_{h}^{\text {graph }} & :=\Gamma_{h} \backslash\left(\Gamma_{h}^{\text {jump }} \cup \Gamma_{h}^{\text {cut }}\right) .
\end{aligned}
$$

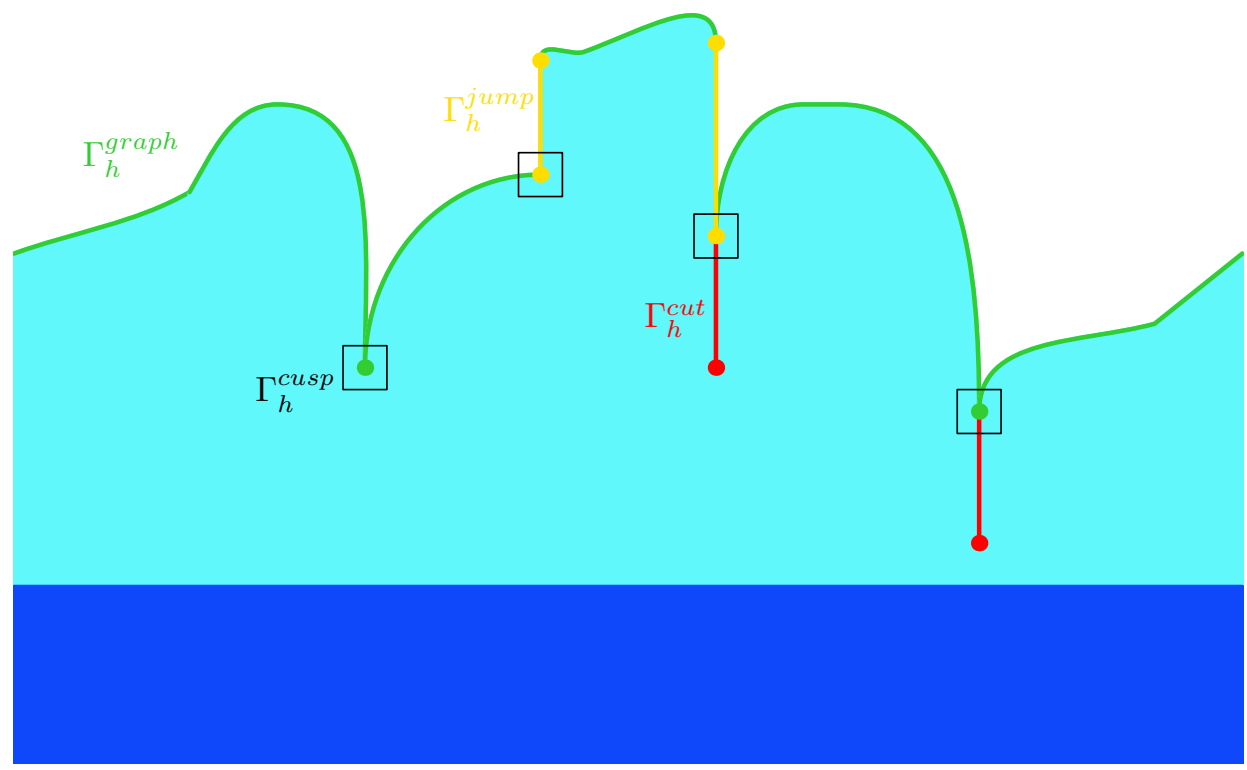

FIgURE 2. In the figure above an admissible profile function $h$ is displayed. The portions of $\Gamma_{h}$ corresponding to $\Gamma_{h}^{\text {graph }}, \Gamma_{h}^{\text {jump }}$, and $\Gamma_{h}^{c u t}$ are represented with the colors green, yellow, and red respectively. The points in $\Gamma_{h}^{\text {cusp }}$ are marked by enclosing them within squares.

We observe that $\Gamma_{h}^{\text {graph }}$ represents the regular part of the graph of $h$, whilst both $\Gamma_{h}^{j u m p}$ and $\Gamma_{h}^{c u t}$ consist in (at most countable) unions of segments, corresponding to the jumps and the cuts in the graph of $h$, respectively (see Figure 2). Notice also that

$$
\Gamma_{h}=\tilde{\Gamma}_{h} \sqcup \Gamma_{h}^{c u t} .
$$

Let us also identify the set of cusps in $\Gamma_{h}$ by $\Gamma_{h}^{c u s p}:=\left\{\left(x, h^{-}(x)\right):\right.$ either $x \in J(h)$

$$
\text { or we have that } \left.x \notin J(h) \text { with } h_{+}^{\prime}(x)=+\infty \text { or } h_{-}^{\prime}(x)=-\infty\right\}
$$

(see Figure 2).

For every $h \in A P(a, b)$ we indicate its set of of zeros by

$$
Z_{h}:=\Gamma_{h} \cap\{x \in[a, b]: h(x)=0\} .
$$


For every $x \in Z_{h}$, let $\theta^{ \pm}(x)$ be the internal angles, with amplitude smaller or equal to $\frac{\pi}{2}$, between the $x$-axis and the tangents to $\Gamma_{h}$ in $(x, 0)$ from the left and from the right, with slopes $h_{-}^{\prime}(x)$ and $h_{+}^{\prime}(x)$, respectively. Consider the set

$$
I_{h}:=\left\{(c, d) \subset Z_{h}: c<d \text { and } c, d \notin \operatorname{Int}\left(Z_{h}\right)\right\},
$$

and let

$$
P_{h}:=Z_{h} \backslash \bigcup_{(c, d) \in I_{h}}[c, d]
$$

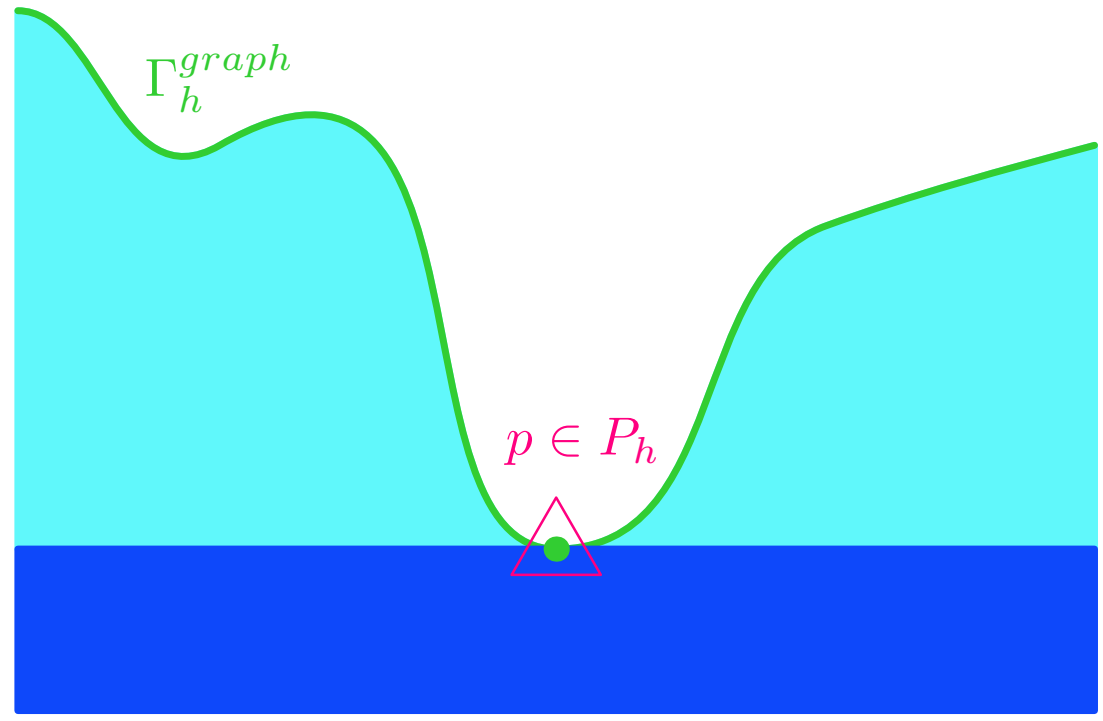

Figure 3. A valley at an isolated point $p \in P_{h}$ is displayed. The point $p$ is indicated by enclosing it in a pink triangle

We will refer to the endpoints $c$ and $d$ of any interval $(c, d) \in I_{h}$ as borders of (two different) islands and to the points in $P_{h}$ as valleys, and we observe that

$$
\begin{cases}\theta^{-}(x)=0 & \text { for every } x \in(c, d] \\ \theta^{+}(x)=0 & \text { for every } x \in[c, d)\end{cases}
$$

(see Figures 3 and 4).

We now define the family $X$ of admissible film configurations as

$$
X:=\left\{(u, h): u \in H_{\mathrm{loc}}^{1}\left(\Omega_{h} ; \mathbb{R}^{2}\right) \text { and } h \in A P(a, b)\right\}
$$

and we endow $X$ with the following notion of convergence.

Definition 2.1. We say that a sequence $\left\{\left(u_{n}, h_{n}\right)\right\} \subset X$ converges to $(u, h) \in X$, and we write $\left(u_{n}, h_{n}\right) \rightarrow(u, h)$ in $X$ if

1. $\sup _{n} \operatorname{Var} h_{n}<+\infty$,

2. $\mathbb{R}^{2} \backslash \Omega_{h_{n}}$ converges to $\mathbb{R}^{2} \backslash \Omega_{h}$ in the Hausdorff metric,

3. $u_{n} \rightarrow u$ weakly in $H^{1}\left(\Omega^{\prime} ; \mathbb{R}^{2}\right)$ for every $\Omega^{\prime} \subset \subset \Omega_{h}$. 


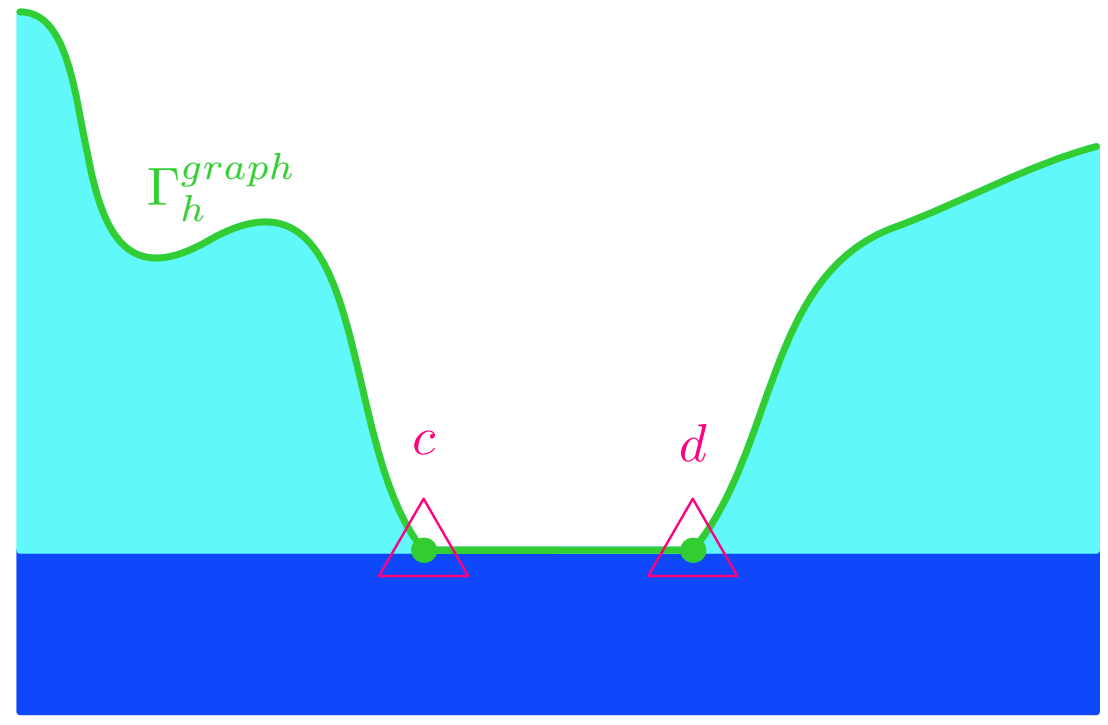

Figure 4. An interval $(c, d) \in I_{h}$ is displayed. The points $c, d$ indicated with pink triangles are the only ones in $I_{h}$ with non-trivial contact angles.

Let us also consider the following subfamily $X_{\text {Lip }}$ in $X$ of configurations with Lipschitz profiles, namely,

$$
X_{\text {Lip }}:=\left\{(u, h): u \in H_{\text {loc }}^{1}\left(\Omega_{h} ; \mathbb{R}^{2}\right), h \text { is Lipschitz }\right\} .
$$

We recall from Subsection 1.2 that the thin-film model analyzed in this paper is characterized by the energy $\mathcal{F}$ defined by (1.2) on configurations $(u, h) \in X$.

We state here the definition of $\mu$-local minimizers of the energy $\mathcal{F}$.

Definition 2.2. We say that a pair $(u, h) \in X$ is a $\mu$-local minimizer of the functional $\mathcal{F}$ if $\mathcal{F}(u, h)<+\infty$ and there exists $\mu>0$ such that

$$
\mathcal{F}(u, h) \leq \mathcal{F}(v, g)
$$

for every $(v, g) \in X$ satisfying $\left|\Omega_{g}^{+}\right|=\left|\Omega_{h}^{+}\right|$and $\left|\Omega_{g} \Delta \Omega_{h}\right| \leq \mu$.

Note that every global minimizer (with or without volume constraint) is a $\mu$-local minimizer.

2.2. Statement of the main results. The paper contains two main theorems. Consider the situation in which $\mathbb{C}_{f}$ and $\mathbb{C}_{s}$ are the elasticity tensors of isotropic materials with Lamé coefficients $\mu_{f}, \lambda_{f}$, and $\mu_{s}, \lambda_{s}$, respectively.

Our first result regards the identification of contact angle conditions for the $\mu$-local minimizers $(u, h) \in X$ of $\mathcal{F}$.

Theorem 2.3 (Contact-angle conditions). Assume that the Lamé coefficients of the film and the substrate satisfy

$$
\mu_{s} \geq \mu_{f}>0 \quad \text { and } \quad \mu_{s}+\lambda_{s} \geq \mu_{f}+\lambda_{f}>0 .
$$

Then, every $\mu$-local minimizer $(u, h) \in X$ of $\mathcal{F}$ satisfies the following properties: 
1. For every $p, c, d \in Z_{h} \backslash\left(\Gamma_{h}^{c u s p} \cup \Gamma_{h}^{c u t}\right)$ such that $p \in P_{h}$ and $(c, d) \in I_{h}$ we have

$$
\theta^{-}(p)=\theta^{+}(p)=\theta^{-}(c)=\theta^{+}(d)=\arccos (\beta),
$$

where

$$
\beta:=\frac{\min \left\{\gamma_{f}, \gamma_{s}-\gamma_{f s}\right\}}{\gamma_{f}} .
$$

2. If $\beta<1$, then $P_{h} \backslash\left(\Gamma_{h}^{\text {cusp }} \cup \Gamma_{h}^{c u t}\right)=\emptyset$.

3. If $\beta \neq 0$, then $\Gamma_{h}^{\text {jump }} \cap Z_{h}=\emptyset$.

We remark that Theorem 2.3 is the analytical validation of the Young-Dupré law for angles not greater than $\pi / 2$. Let us sum up here the possible scenarios for the wetting angles:

Wetting regime: For $\gamma_{s}-\gamma_{f s} \geq \gamma_{f}$ all contact angles of $\Gamma_{h} \backslash\left(\Gamma_{h}^{c u t} \cup \Gamma_{h}^{c u s p}\right)$ are zero.

Dewetting regime: For $\gamma_{s}-\gamma_{f s}<\gamma_{f}$ all nontrivial contact angles $\theta$ of points in $Z_{h} \backslash\left(\Gamma_{h}^{c u t} \cup \Gamma_{h}^{c u s p}\right)$ are such that

$$
\cos \theta=\frac{\gamma_{s}-\gamma_{f s}}{\gamma_{f}}
$$

We stress that, in agreement with the Young-Dupré law, jumps at island borders (see Figure 5) are excluded when $\min \left\{\gamma_{f}, \gamma_{s}-\gamma_{f s}\right\} / \gamma_{f} \neq 0$. Note also that the contact angles

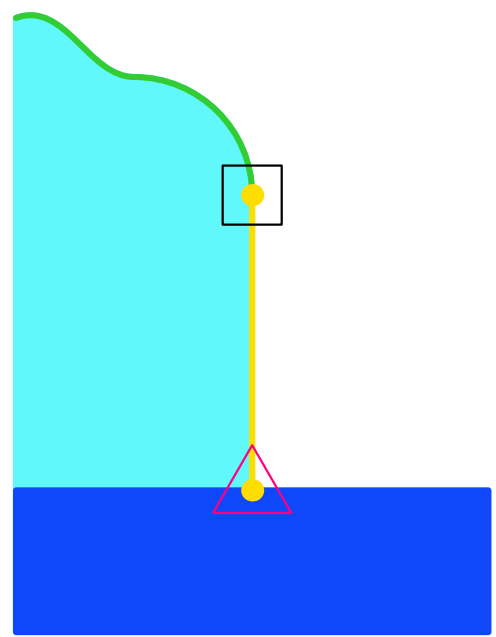

FiguRE 5. Example of a jump at an island border (here indicated with a pink triangle). This is the only type of jump allowed by Theorem 2.3 and only if $\min \left\{\gamma_{f}, \gamma_{s}-\gamma_{f s}\right\} / \gamma_{f}=0$.

at valleys are always zero (and there are no jumps at valleys), since valleys exist only for the wetting regime when $\beta=1$.

However, our analysis allows the set $D_{h}:=\left[\left(\Gamma_{h}^{c u s p} \cup \Gamma_{h}^{c u t}\right) \backslash \Gamma_{h}^{j u m p}\right] \cap Z_{h}$ to be nonempty. It seems though that this is not a restriction of our method but it is in agreement with the experimental evidence. Points in $D_{h}$ may represent in fact dislocations that are experimentally shown to form as a further mode of strain relief and 
to migrate at the film/substrate interface. We kindly refer the reader to [13] and the reference therein for more details on dislocations in epitaxy and for a thin-film model accounting for their presence. Some examples of contact angles in $D_{h}$ are displayed in Figure 6 .
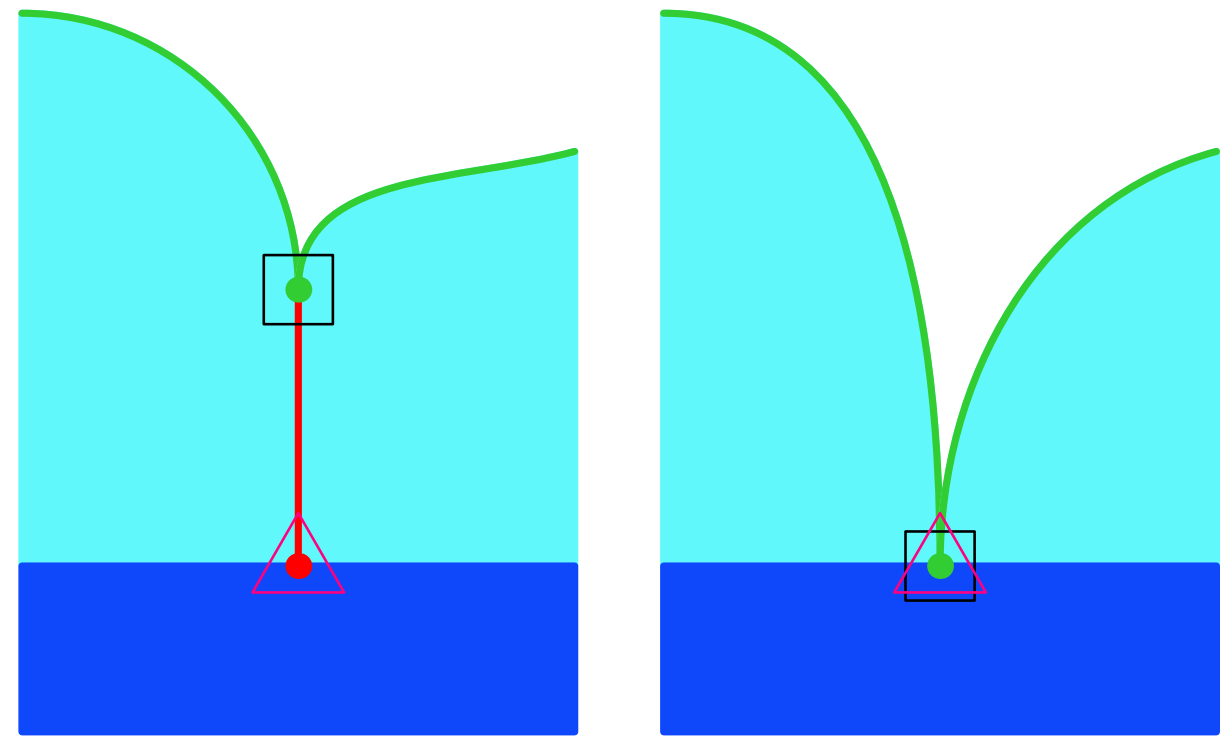

Figure 6. Cuts (left) and cusps (right) may represents dislocations at the film/substrate interface.

Regarding condition (4.2), assuming $\mu_{f}, \mu_{s}>0, \lambda_{f}+\mu_{f}>0$, and $\lambda_{s}+\mu_{s}>0$ guarantees the ellipticity of the transmission problem associated to the Euler-Lagrange equations of $\mu$-local minimizers of $\mathcal{F}$ (see [20, Lemma 1.3]). The assumption

$$
\mu_{s} \geq \mu_{f} \quad \text { and } \quad \mu_{s}+\lambda_{s} \geq \mu_{f}+\lambda_{f}
$$

is a quasi-monotonicity condition. This kind of assumptions are classically considered in transmission problems for elliptic systems, we refer the reader to [9] for the first formulation for transmission problems with the Laplace operator (see also [21] and the references therein). As stated in [22] where (2.8) is introduced, "it seems that the quasimonotonicity condition [...] describes a class of composites which can sustain higher loads before breaking". Furthermore, condition (2.8) implies that the shear and the $P$-wave moduli of the substrate are higher than those of the film. As such parameters are elastic moduli for the materials, this entails that the substrate is stiffer than the film. Such requirement appears to be natural in the thin-film models here considered from [29], where only the boundary of the film and not the boundary of the substrate is allowed to deform. We recall that in these models the film/substrate interface is forced to coincide with the $x$-axis. As a matter of fact, quasi-monotonicity conditions are strongly related to the particular geometry in which the transmission problem is considered, and in particular to the position of the transmission interface at boundary corners. Other conditions than (2.8) might be included if the film/substrate interface is not maintained fixed as in [29]. 
The final main theorem of the paper concerning the regularity of optimal profiles is the following.

Theorem 2.4 (Regularity). Assume that the Lamé coefficients of the film and the substrate satisfy (4.2).

Then, every $\mu$-local minimizer $(u, h) \in X$ of $\mathcal{F}$ has the following regularity properties:

1. Cusps points and vertical cuts are at most finite;

2. $\Gamma_{h}^{r e g}:=\Gamma_{h} \backslash\left(\Gamma_{h}^{c u t} \cup \Gamma_{h}^{\text {cusp }}\right)$ is locally the graph of a Lipschitz function;

3. $\Gamma_{h}^{r e g} \backslash Y_{h}$ is $C^{1, \alpha}$ for all $\alpha \in(0,1 / 2)$, where $Y_{h}$ is the subset of $Z_{h} \cap \Gamma_{h}^{\text {reg }}$ containing points with nonzero contact angles for $h$;

4. The set

$$
A_{h}:= \begin{cases}\Gamma_{h}^{r e g} \backslash Z_{h} & \text { if } \mathbb{C}_{f} \neq \mathbb{C}_{s} \\ \Gamma_{h}^{r e g} \backslash Y_{h} & \text { if } \mathbb{C}_{f}=\mathbb{C}_{s}\end{cases}
$$

is analytic and satisfies the Euler-Lagrange equation

$$
\gamma_{f} k_{A_{h}}=\tau_{A_{h}}\left(W_{0}\left(\cdot, E u(\cdot)-E_{0}\right)\right)+\lambda_{0} \text { on } A_{h},
$$

where the function $k_{A_{h}}(\cdot)$ denotes the curvature of $A_{h}, \tau_{A_{h}}(\cdot)$ is the trace operator on $A_{h}$, and $\lambda_{0}$ is a suitable Lagrange multiplier.

We also point out that for $\mathbb{C}_{f}=\mathbb{C}_{s}$ in view of Assertion 5. of Theorem 2.4 for every $\mu$-local minimizer $(u, h)$ of $\mathcal{F}$ the set $Z_{h}$ has either finite cardinality or nonempty interior in the $x$-axis. Finally, we observe that in the wetting regime for $\mathbb{C}_{f}=\mathbb{C}_{s}$ the analytic portion of the graph $A_{h}$ coincides with $\Gamma_{h}^{r e g}$ since by the assertions 1 . and 2. of Theorem 2.3 we have $Y_{h}=\emptyset$.

Remark 2.5. The results in Theorem 2.3 hold also for $\mu$-local minimizers of the energy

$$
\begin{aligned}
\mathcal{E}\left(u^{+}, u^{-}, h\right):= & \int_{\Omega_{h}^{+}} \mathbb{C}_{f} E u^{+}(x, y): E u^{+}(x, y) d x d y \\
& +\int_{\Omega_{h}^{-}} \mathbb{C}_{s} E u^{-}(x, y): E u^{-}(x, y) d x d y+\int_{\Gamma_{h}} \varphi(y) d \mathcal{H}^{1} \\
& +\gamma_{f s}(b-a)+2 \gamma_{f} \mathcal{H}^{1}\left(\Gamma_{h}^{c u t}\right)
\end{aligned}
$$

for every $\left(u^{+}, u^{-}, h\right) \in \tilde{X}$, where

$$
\begin{array}{r}
\tilde{X}:=\left\{\left(u^{+}, u^{-}, h\right): u^{+} \in H_{\mathrm{loc}}^{1}\left(\Omega_{h}^{+} ; \mathbb{R}^{2}\right), u^{-} \in H_{\mathrm{loc}}^{1}\left(\Omega_{h}^{-} ; \mathbb{R}^{2}\right),\right. \\
\left.u^{+}(\cdot, 0)-u^{-}(\cdot, 0)=\left(e_{0}, 0\right), \text { and } h \in A P(a, b)\right\} .
\end{array}
$$

In fact, there is a 1-1 correspondence between triples $\left(u^{+}, u^{-}, h\right)$ that are $\mu$-local minimizers of $(2.10)$, and pairs $(u, h)$ which are $\mu$-local minimizers of $(1.2)$, with

$$
u(x, y):= \begin{cases}u^{+}(x, y)-\left(e_{0} x, 0\right) & \text { if } y \geq 0 \\ u^{-}(x, y) & \text { if } y<0\end{cases}
$$

for $(x, y) \in \Omega_{h}$. Energy functionals similar to (2.10) are considered for the corresponding evolution problem (see, e.g., [34]). 


\section{Properties OF LOCAL MiNIMIZERS}

In this section we start analyzing the regularity of $\mu$-local minimizers $(u, h)$ of $(1.2)$. In the first subsection we recall the results in [7], showing that optimal profiles $h$ satisfy the internal-ball condition. The second subsection is devoted to establish a decay estimate for the minimizing displacements $u$, and relies on some techniques introduced in the setting of transmission problems for elliptic systems (see [20, 21, 25, 26]).

3.1. Internal-ball condition. In this subsection we collect some first regularity results for local minimizers. We refer to [7] for the proofs of the next two propositions. The first observation is that the area constraint in the minimization problem of Definition 2.2 can be replaced with a suitable penalization in the energy functional.

Proposition 3.1. Let $(u, h) \in X$ be a $\mu$-local minimizer for the functional $\mathcal{F}$. Then there exists $\lambda_{0}>0$ such that

$$
\mathcal{F}(u, h)=\min \left\{\mathcal{F}(v, g)+\lambda|| \Omega_{h}^{+}|-| \Omega_{g}^{+} \|:(v, g) \in X,\left|\Omega_{g} \Delta \Omega_{h}\right| \leq \frac{\mu}{2}\right\}
$$

for all $\lambda \geq \lambda_{0}$.

We are now ready to recall the internal-ball condition for optimal profiles.

Proposition 3.2 (Internal-ball condition). Let $(u, h) \in X$ be a $\mu$-local minimizer for the functional $\mathcal{F}$. Then, there exists $\rho_{0}>0$ such that for every $z \in \bar{\Gamma}_{h}$ we can choose a point $P_{z}$ for which $B\left(P_{z}, \rho_{0}\right) \cap((a, b) \times \mathbb{R}) \subset \Omega_{h}$, and

$$
\partial B\left(P_{z}, \rho_{0}\right) \cap \bar{\Gamma}_{h}=\{z\} .
$$

We point out that in view of Proposition 3.2 the upper-end point of each cut is a cusp point (see Figure 2).

The following proposition is a consequence of the internal-ball condition.

Proposition 3.3. Let $(u, h) \in X$ be a $\mu$-local minimizer for the functional $\mathcal{F}$. Then for any $z_{0} \in \bar{\Gamma}_{h}$ there exist an orthonormal basis $\mathbf{v}_{1}, \mathbf{v}_{2} \in \mathbb{R}^{2}$, and a rectangle

$$
Q:=\left\{z_{0}+s \mathbf{v}_{1}+t \mathbf{v}_{2}:-a^{\prime}<s<a^{\prime},-b^{\prime}<t<b^{\prime}\right\}
$$

$a^{\prime}, b^{\prime}>0$, such that $\Omega_{h} \cap Q$ has one of the following two representations:

1. There exists a Lipschitz function $g:\left(-a^{\prime}, a^{\prime}\right) \rightarrow\left(-b^{\prime}, b^{\prime}\right)$ such that $g(0)=0$ and

$$
\Omega_{h} \cap Q:=\left\{z_{0}+s \mathbf{v}_{1}+t \mathbf{v}_{2}:-a^{\prime}<s<a^{\prime},-b^{\prime}<t<g(s)\right\} \cap((a, b) \times \mathbb{R}) .
$$

In addition, the function $g$ admits left and right derivatives at all points that are, respectively, left and right continuous.

2. There exist two Lipschitz functions $g_{1}, g_{2}:\left[0, a^{\prime}\right) \rightarrow\left(-b^{\prime}, b^{\prime}\right)$ such that $g_{i}(0)=$ $\left(g_{i}\right)_{+}^{\prime}(0)=0$ for $i=1,2, g_{1} \leq g_{2}$, and

$$
\Omega_{h} \cap Q:=\left\{z_{0}+s \mathbf{v}_{1}+t \mathbf{v}_{2}: 0<s<a^{\prime},-b^{\prime}<t<g_{1}(s) \text { or } g_{2}(s)<t<b^{\prime}\right\} .
$$

In addition, the functions $g_{1}, g_{2}$ admit left and right derivatives at all points that are, respectively, left and right continuous.

For the proof of Proposition 3.3 we refer the reader to [6, Lemma 3] and [12, Proposition 3.5]. In particular Proposition 3.3 entails that the set

$$
\Gamma_{h}^{r e g}=\Gamma_{h} \backslash\left(\Gamma_{h}^{c u s p} \cup \Gamma_{h}^{c u t}\right)
$$

is locally Lipschitz (see the proof of Theorem 2.4 in Section 5 for more details). 
3.2. Decay estimate. From now on we work under the assumption that both the film and the substrate are made of linearly elastic isotropic materials, and we denote by $\mu_{f}$, $\lambda_{f}, \mu_{s}, \lambda_{s}$ their Lamé coefficients. Note that

$$
\mathbb{C}_{\sigma} E u=2 \mu_{\sigma} E u+\lambda_{\sigma}(\operatorname{div} u) I d, \quad \sigma=f, s,
$$

for every $u \in H^{1}\left(\Omega_{h} ; \mathbb{R}^{2}\right)$.

In order to prove the decay estimate of Proposition 3.6 for minimizing configurations $(u, h)$ at the points of $\Gamma_{h}^{r e g}$ a blow-up around such points is needed. As the graph is allowed to touch the film/substrate interface, we are lead to consider transmission problems for Lamé systems in conical sets. We first state a preliminary lemma, relying on [19, Theorem 1.5.2.8], and whose proof is contained in [12, Lemma 3.12]).

Lemma 3.4. Let $\mathcal{C}$ be a circular sector of amplitude $\theta \in(0,2 \pi)$ and radius $R>0$. Assume that $\mathcal{C}$ is the reference configuration of a linearly elastic isotropic material whose Lamé coefficients are denoted by $\mu$ and $\lambda$. Let $g \in H^{1 / 2}\left(\partial \mathcal{C} ; \mathbb{R}^{2}\right)$ be a function vanishing in a neighborhood of the origin. Then there exists a function $v \in H^{2}\left(\mathcal{C} ; \mathbb{R}^{2}\right)$ such that

$$
[2 \mu E v+\lambda(\operatorname{div} v) I d] \nu_{\mathcal{C}}=g \text { on } \partial \mathcal{C},
$$

where $\nu_{\mathcal{C}}$ is the outer unit normal to $\mathcal{C}$ (where it exists), and

$$
v=0 \quad \text { on } \partial \mathcal{C} .
$$

In the following proposition we assess the regularity of weak solutions to transmission problems for Lamé systems in conical sets.

Proposition 3.5. Let $\mathcal{C}$ be the set given by

$$
\mathcal{C}:=\operatorname{Int}\left(\bigcup_{i=1}^{3} \overline{\mathcal{C}_{i}}\right)
$$

where $\mathcal{C}_{i}, i=1,2,3$, are the circular sectors defined by

$$
\mathcal{C}_{i}:=\left\{(x, y): x=\rho \cos (\theta), y=\rho \sin (\theta), \text { with } 0<\rho<R, \text { and } \theta_{i-1}<\theta<\theta_{i}\right\}
$$

with $R>0$, and $0=: \theta_{0} \leq \theta_{1}<\theta_{2} \leq \theta_{3}<2 \pi$ (see Figure 7). Denote by

$$
\Gamma_{1,0}:=(0, R)
$$

and

$$
\Gamma_{3,0}:=\left\{\left(\rho \cos \left(\theta_{3}\right), \rho \sin \left(\theta_{3}\right)\right) \in \mathbb{R}^{2} \text { with } 0<\rho<R\right\},
$$

the two external sides of $\mathcal{C}$, and by

$$
\Gamma_{i}:=\left\{(x, y): x=R \cos \theta, y=R \sin \theta, \text { with } \theta_{i-1} \leq \theta<\theta_{i}\right\},
$$

for $i=1,2,3$ the curvilinear portions of its boundary. Finally, consider the transmission interfaces

$$
\Gamma_{i, i+1}:=\partial \mathcal{C}_{i} \cap \partial \mathcal{C}_{i+1} \quad \text { for } i=1,2 .
$$

We assume that each set $\mathcal{C}_{i}$ is the reference configuration of a linearly elastic, isotropic material whose Lamé coefficients are denoted by $\mu_{i}$ and $\lambda_{i}$, with $\mu_{3}:=\mu_{1}$ and $\lambda_{3}:=\lambda_{1}$, and satisfy the quasi-monotonicity condition:

$$
\mu_{2} \geq \mu_{1}>0 \text { and } \mu_{2}+\lambda_{2} \geq \mu_{1}+\lambda_{1}>0 .
$$




$$
\begin{aligned}
& \text { Let }\left(u_{1}, u_{2}, u_{3}\right) \in \prod_{i=1}^{3} H^{1}\left(\mathcal{C}_{i} ; \mathbb{R}^{2}\right) \text { be a weak solution of the transmission problem: } \\
& \begin{cases}\mu_{i} \Delta u_{i}+\left(\lambda_{i}+\mu_{i}\right) \nabla\left(\operatorname{div} u_{i}\right)=f_{i} & \text { in } \mathcal{C}_{i}, i=1,2,3, \\
{\left[2 \mu_{i} E u_{i}+\lambda_{i}\left(\operatorname{div} u_{i}\right) I d\right] \nu_{i, 0}=0} & \text { on } \Gamma_{i, 0}, i=1,3, \\
{\left[2 \mu_{i} E u_{i}+\lambda_{i}\left(\operatorname{div} u_{i}\right) I d\right] \nu_{i}=g_{i}} & \text { on } \Gamma_{i}, i=1,2,3, \\
u_{i}-u_{i+1}=0 & \text { on } \Gamma_{i, i+1}, i=1,2, \\
{\left[2 \mu_{i} E u_{i}-2 \mu_{i+1} E u_{i+1}\right.} & \\
\left.\quad+\lambda_{i}\left(\operatorname{div} u_{i}\right) I d-\lambda_{i+1}\left(\operatorname{div} u_{i+1}\right) I d\right] \nu_{i, i+1}=0 & \text { on } \Gamma_{i, i+1}, i=1,2,\end{cases}
\end{aligned}
$$

where the data $f_{i}$ and $g_{i}$ satisfy $f_{i} \in L^{2}\left(\mathcal{C}_{i}\right), g_{i} \in H^{1 / 2}\left(\Gamma_{i}, \mathbb{R}^{2}\right), i=1,2,3$, the vectors $\nu_{i, i+1}$ are the normal to $\Gamma_{i, i+1}$ external to $\mathcal{C}_{i}, i=1,2$, and the vectors $\nu_{1,0}, \nu_{3,0}$, and $\nu_{i}$ are the outer unit normals to $\Gamma_{1,0}, \Gamma_{3,0}$, and $\Gamma_{i}, i=1,2,3$, respectively.

If there exists a vector $\tau \in \mathbb{R}^{2} \backslash\{0\}$ such that $\tau \in \mathcal{C}_{2}$ and $-\tau \notin \overline{\mathcal{C}}$, then there exists a neighbourhood $U$ of the origin such that

$$
u \in H^{3 / 2+\varepsilon}\left(U \cap \mathcal{C}_{i}\right)
$$

for some $\varepsilon>0$ and for $i=1,2,3$.

Proof. Let $\varphi \in C_{c}^{\infty}(\mathcal{C})$ be a cut-off function such that $0 \leq \varphi \leq 1$ in $\mathcal{C}$, with $\operatorname{supp} \varphi \subset \subset B_{R / 2}$, and $\varphi \equiv 1$ in $B_{R / 3}$, where here $B_{R / 2}$ and $B_{R / 3}$ are the balls centered in the origin and with radii $R / 2$ and $R / 3$, respectively. Consider the maps $\left(\tilde{u}_{1}, \tilde{u}_{2}, \tilde{u}_{3}\right) \in \prod_{i=1}^{3} H^{1}\left(\mathcal{C}_{i} ; \mathbb{R}^{2}\right)$, defined as $\tilde{u}_{i}:=\varphi u_{i}, i=1,2,3$. By straightforward computation, and in view of $(3.19)$, the triple $\left(\tilde{u}_{1}, \tilde{u}_{2}, \tilde{u}_{3}\right)$ solves the transmission problem

$$
\begin{cases}\mu_{i} \Delta \tilde{u}_{i}+\left(\lambda_{i}+\mu_{i}\right) \nabla\left(\operatorname{div} \tilde{u}_{i}\right)=\tilde{f}_{i} & \text { in } \mathcal{C}_{i}, i=1,2,3, \\ {\left[2 \mu_{i} E \tilde{u}_{i}+\lambda_{i}\left(\operatorname{div} \tilde{u}_{i}\right) I d\right] \nu_{i, 0}=\tilde{g}_{i}} & \text { on } \Gamma_{i, 0}, i=1,3, \\ {\left[2 \mu_{i} E \tilde{u}_{i}+\lambda_{i}\left(\operatorname{div} \tilde{u}_{i}\right) I d\right] \nu_{i}=0} & \text { on } \Gamma_{i}, i=1,2,3, \\ \tilde{u}_{i}-\tilde{u}_{i+1}=0 & \text { on } \Gamma_{i, i+1}, i=1,2, \\ {\left[2 \mu_{i} E \tilde{u}_{i}-2 \mu_{i+1} E \tilde{u}_{i+1}\right.} & \\ \left.\quad+\lambda_{i}\left(\operatorname{div} \tilde{u}_{i}\right) I d-\lambda_{i+1}\left(\operatorname{div} \tilde{u}_{i+1}\right) I d\right] \nu_{i, i+1}=\tilde{h}_{i} & \text { on } \Gamma_{i, i+1}, i=1,2,\end{cases}
$$

where $\tilde{f}_{i} \in L^{2}\left(\mathcal{C}_{i}\right), \tilde{g}_{i} \in H^{1 / 2}\left(\Gamma_{i, 0} ; \mathbb{R}^{2}\right)$, and $\tilde{h}_{i} \in H^{1 / 2}\left(\Gamma_{i, i+1} ; \mathbb{R}^{2}\right)$ for every $i$, and the maps $\tilde{g}_{i}$ and $\tilde{h}_{i}$ vanish in the intersection of their domains with $B_{R / 3}$. By applying Lemma 3.4 to both sets $\mathcal{C}_{i}, i=1,3$, with

$$
g= \begin{cases}\tilde{g}_{i} & \text { on } \Gamma_{i, 0}, \\ 0 & \text { on } \Gamma_{i}, \\ \tilde{h}_{i} & \text { on } \Gamma_{i, i+1},\end{cases}
$$

we obtain functions $v_{i} \in H^{2}\left(\mathcal{C}_{i} ; \mathbb{R}^{2}\right)$ such that

$$
\left[2 \mu E v_{i}+\lambda\left(\operatorname{div} v_{i}\right) I d\right] \nu_{\mathcal{C}_{i}}=g \quad \text { on } \partial \mathcal{C}_{i},
$$

where $\nu_{\mathcal{C}_{i}}$ is the outer unit normal to $\mathcal{C}_{i}$ (where it exists), and

$$
v_{i}=0 \quad \text { on } \partial \mathcal{C}_{i}
$$


Setting $\left(w_{1}, w_{2}, w_{3}\right):=\left(\tilde{u}_{1}-v_{1}, \tilde{u}_{2}, \tilde{u}_{3}-v_{3}\right) \in \prod_{i=1}^{3} H^{1}\left(\mathcal{C}_{i} ; \mathbb{R}^{2}\right)$, by $(3.3)$, and $(3.4)-(3.6)$ there holds

$$
\begin{cases}\mu_{i} \Delta w_{i}+\left(\lambda_{i}+\mu_{i}\right) \nabla\left(\operatorname{div} w_{i}\right)=\hat{f}_{i} & \text { in } \mathcal{C}_{i}, i=1,2,3, \\ {\left[2 \mu_{i} E w_{i}+\lambda_{i}\left(\operatorname{div} w_{i}\right) I d\right] \nu_{i, 0}=0} & \text { on } \Gamma_{i, 0}, i=1,3, \\ {\left[2 \mu_{i} E w_{i}+\lambda_{i}\left(\operatorname{div} w_{i}\right) I d\right] \nu_{i}=0} & \text { on } \Gamma_{i}, i=1,2,3, \\ w_{i}-w_{i+1}=0 & \text { on } \Gamma_{i, i+1}, i=1,2, \\ {\left[2 \mu_{i} E w_{i}-2 \mu_{i+1} E w_{i+1}\right.} & \\ \left.\quad+\lambda_{i}\left(\operatorname{div} w_{i}\right) I d-\lambda_{i+1}\left(\operatorname{div} w_{i+1}\right) I d\right] \nu_{i, i+1}=0 & \text { on } \Gamma_{i, i+1}, i=1,2,\end{cases}
$$

where $\hat{f}_{i} \in L^{2}\left(\mathcal{C}_{i}\right)$ for $i=1,2,3$. By [22, Theorem 2] we obtain that there exists a neighborhood $\tilde{U}$ of the origin such that $w_{i} \in H^{3 / 2+\varepsilon}\left(\tilde{U} \cap \mathcal{C}_{i}\right)$ for $i=1,2,3$. The thesis follows by observing that on $U:=\tilde{U} \cap B_{R / 3}$, the triple $\left(u_{1}, u_{2}, u_{3}\right)$ satisfies

$$
\left(u_{1}, u_{2}, u_{3}\right)=\left(\tilde{u}_{1}, \tilde{u}_{2}, \tilde{u}_{3}\right)=\left(w_{1}+v_{1}, w_{2}, w_{3}+v_{3}\right),
$$

and by the regularity of the maps $v_{i}, i=1,3$.

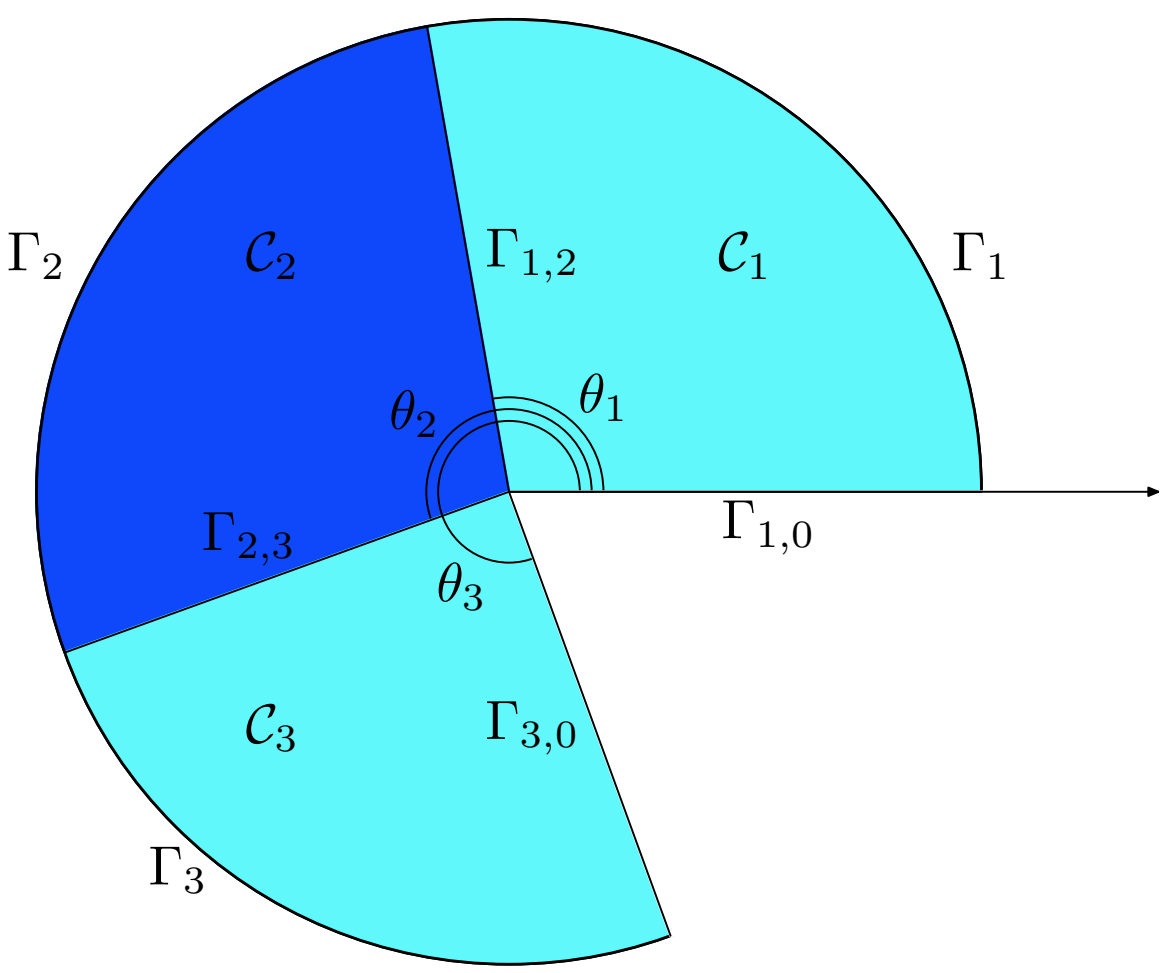

Figure 7. The geometry of the set $\mathcal{C}$ on which we consider the transmission problem of Proposition 3.5 is depicted. The lines $\Gamma_{1,2}$ and $\Gamma_{2,3}$ are transmission interfaces for such problem. 
We are now ready to provide a decay estimate for the gradient of minimizing displacements at the points in which the graph of the corresponding minimizing profile is locally Lipschitz.

Proposition 3.6 (Decay estimate). Let $(u, h) \in X$ be a $\mu$-local minimizer for the functional $\mathcal{F}$ and assume that the Lamé coefficients of film and substrate satisfy the monotonicity condition (4.2). Let $z_{0}:=\left(x_{0}, h\left(x_{0}\right)\right) \in \Gamma_{h} \backslash\left(\Gamma_{h}^{c u t} \cup \Gamma_{h}^{\text {cusp }}\right)$. Then there exists a constant $C>0$, a radius $r_{0}$, and an exponent $\frac{1}{2}<\alpha<1$, such that

$$
\int_{B\left(z_{0}, r\right) \cup \Omega_{h}}|\nabla u|^{2} d x d y \leq C r^{2 \alpha}
$$

for all $0<r<r_{0}$.

Proof. We begin by considering the case in which $h\left(x_{0}\right)=0$. If there exists a constant $C$ such that

$$
\limsup _{r \rightarrow 0} \frac{1}{r^{2}} \int_{B\left(z_{0}, r\right) \cap \Omega_{h}}|\nabla u|^{2} d x d y \leq C,
$$

then there is nothing to prove. Thus, we assume that this does not hold and that there exists a sequence $\left\{r_{n}\right\} \subset \mathbb{R}$ such that $r_{n} \rightarrow 0$ and

$$
\limsup _{n \rightarrow+\infty} \frac{1}{r_{n}^{2}} \int_{B\left(z_{0}, r_{n}\right) \cap \Omega_{h}}|\nabla u|^{2} d x d y=+\infty .
$$

We subdivide the proof into three steps.

Step 1: We claim that there exist an orthonormal basis $\left\{v_{1}, v_{2}\right\}$ of $\mathbb{R}^{2}$, three constants $C_{1}, L>0, \tau_{0} \in(0,1)$, and an exponent $\frac{1}{2}<\beta<1$ such that for all $\tau \in\left(0, \tau_{0}\right)$ there exists a radius $0<r_{\tau}<1$ satisfying

$$
\int_{C\left(z_{0}, \tau r_{n}\right)}|\nabla u|^{2} d x d y \leq C_{1} \tau^{2 \beta} \int_{C\left(z_{0}, r_{n}\right)}\left(1+|\nabla u|^{2}\right) d x d y,
$$

for all $0<r_{n}<r_{\tau}$, where

$$
C\left(z_{0}, r_{n}\right):=\Omega_{h} \cap\left\{z_{0}+s v_{1}+t v_{2}:-r_{n}<s<r_{n},-4 L r_{n}<t<4 L r_{n}\right\} .
$$

We point out that, once claim (3.9) is proved, the assert of the theorem follows arguing as in [12, Theorem 3.13, Step 6].

To prove (3.9) we first observe that, since $z_{0} \in \Gamma_{h}^{r e g}$, we can apply Proposition 3.3 to obtain a Lipschitz function $g:\left(-a^{\prime}, a^{\prime}\right) \rightarrow\left(-b^{\prime}, b^{\prime}\right)$ with Lip $g \leq L$ for some $L>1$ such that $g(0)=0$, and

$$
\Omega_{h} \cap Q=\left\{z_{0}+s v_{1}+t v_{2}:-a^{\prime}<s<a^{\prime},-b^{\prime}<t<g(s)\right\},
$$

where

$$
Q=\left\{z_{0}+s v_{1}+t v_{2}:-a^{\prime}<s<a^{\prime},-b^{\prime}<t<b^{\prime}\right\} .
$$

Note that $g$ has left (right) derivative in every point that is left (right) continuous. By Korn inequality in Lipschitz domains we deduce that $u \in H^{1}\left(\Omega_{h} \cap Q ; \mathbb{R}^{2}\right)$. If $r_{n} \leq$ $\min \left\{a^{\prime}, \frac{b^{\prime}}{4 L}\right\}$, then $C\left(z_{0}, r_{n}\right) \subset Q \cap \Omega_{h}$. Therefore,

$$
C\left(z_{0}, r_{n}\right)=\left\{z_{0}+s v_{1}+t v_{2}:-r_{n}<s<r_{n},-4 L r_{n}<t<g(s)\right\} .
$$


Fix $C_{1}>0, \tau_{0} \in(0,1)$ and $\beta>\frac{1}{2}$ to be determined later, and assume by contradiction that (3.9) is false for some $\tau \in\left(0, \tau_{0}\right)$. Up to the extraction of a (non-relabeled) subsequence there holds

$$
\int_{C\left(z_{0}, \tau r_{n}\right)}|\nabla u|^{2} d x d y>C_{1} \tau^{2 \beta} \int_{C\left(z_{0}, r_{n}\right)}\left(1+|\nabla u|^{2}\right) d x d y
$$

for a sequence $r_{n} \rightarrow 0$. Define the sets

$$
C_{n}:=\frac{1}{r_{n}}\left(-z_{0}+C\left(z_{0}, r_{n}\right)\right)=\left\{s v_{1}+t v_{2}:-1<s<1,-4 L<t<\frac{g\left(r_{n} s\right)}{r_{n}}\right\} .
$$

We have

$$
\chi_{C_{n}} \rightarrow \chi_{C_{\infty}} \text { a.e. in } \mathbb{R}^{2},
$$

where

$$
C_{\infty}:=\left\{s v_{1}+t v_{2}:-1<s<1,-4 L<t<g_{\infty}(s)\right\}
$$

the function $g_{\infty}$ is defined as

$$
g_{\infty}(s):= \begin{cases}g_{-}^{\prime}(0) s & \text { for } s<0, \\ g_{+}^{\prime}(0) s & \text { for } s>0,\end{cases}
$$

and $\chi_{C_{n}}$ and $\chi_{C_{\infty}}$ are the characteristic functions of the sets $C_{n}$ and $C_{\infty}$, respectively.

Define the maps

$$
u_{n}(z):=\frac{u\left(z_{0}+r_{n} z\right)-a_{n}}{\lambda_{n} r_{n}}, \quad \text { for every } z \in C_{n},
$$

where

$$
a_{n}:=\frac{1}{\left|C\left(z_{0}, r_{n}\right)\right|} \int_{C\left(z_{0}, r_{n}\right)} u(x, y) d x d y, \quad \lambda_{n}^{2}:=\frac{1}{\left|C\left(z_{0}, r_{n}\right)\right|} \int_{C\left(z_{0}, r_{n}\right)}|\nabla u|^{2} d x d y .
$$

We point out that

$$
\frac{1}{\left|C_{n}\right|} \int_{C_{n}}\left|\nabla u_{n}\right|^{2} d z=\frac{1}{\lambda_{n}^{2}\left|C\left(z_{0}, r_{n}\right)\right|} \int_{C\left(z_{0}, r_{n}\right)}|\nabla u|^{2} d x d y=1
$$

and

$$
\begin{aligned}
\int_{C_{n}} & u_{n} d z=\frac{1}{\lambda_{n} r_{n}} \int_{C_{n}} u\left(z_{0}+r_{n} z\right) d x-\frac{a_{n}\left|C_{n}\right|}{\lambda_{n} r_{n}} \\
= & \frac{1}{\lambda_{n} r_{n}^{3}}\left(\int_{C\left(z_{0}, r_{n}\right)} u d x d y-a_{n}\left|C\left(z_{0}, r_{n}\right)\right|\right)=0 .
\end{aligned}
$$

Extend the maps $u_{n}$ to the rectangle

$$
R:=\left\{s v_{1}+t v_{2}:-1<s<1,-4 L<t<4 L\right\}
$$

so that $u_{n} \in W^{1,2}\left(R ; \mathbb{R}^{2}\right)$. By (3.13) we obtain the uniform bound

$$
\left\|u_{n}\right\|_{W^{1,2}\left(R ; \mathbb{R}^{2}\right)} \leq C\left\|\nabla u_{n}\right\|_{L^{2}\left(C_{n} ; \mathbb{M}^{2 \times 2}\right)} \leq C .
$$

Thus, there exist $u_{\infty} \in W^{1,2}\left(R ; \mathbb{R}^{2}\right)$, and $\lambda_{\infty} \in[0,+\infty]$ such that, up to the extraction of a (non-relabelled) subsequence, there holds

$$
u_{n} \rightarrow u_{\infty} \quad \text { weakly in } W^{1,2}\left(R ; \mathbb{R}^{2}\right),
$$

and

$$
\lambda_{n} \rightarrow \lambda_{\infty}
$$


In addition,

$$
\begin{aligned}
& \frac{1}{r_{n}^{2}} \int_{B\left(x_{0}, r_{n}\right) \cap \Omega_{h}}|\nabla u|^{2} d x d y \leq \frac{1}{r_{n}^{2}} \int_{C\left(x_{0}, r_{n}\right)}|\nabla u|^{2} d x d y=\lambda_{n}^{2} \frac{\left|C\left(z_{0}, r_{n}\right)\right|}{r_{n}^{2}} \\
& \quad=\lambda_{n}^{2}\left|C_{n}\right| \leq 12 L \lambda_{n}^{2} .
\end{aligned}
$$

Hence, by (3.8) we conclude that

$$
\lambda_{\infty}=+\infty
$$

In view of a change of variable, the maps $u_{n}$ satisfy the Euler-Lagrange equations

$$
\int_{C_{n}} E \varphi(z): \mathbb{C}\left(r_{n} z_{2}\right) E u_{n}(z) d z=\frac{1}{\lambda_{n}} \int_{C_{n}} E \varphi(z): \mathbb{C}\left(r_{n} z_{2}\right) E_{0}\left(r_{n} z_{2}\right) d z
$$

for every $\varphi \in C_{0}^{1}\left(R ; \mathbb{R}^{2}\right)$. Thus, by (3.11), (3.14), (3.15), and (3.16) we deduce that

$$
\int_{C_{\infty}} E \varphi(z): \mathbb{C}\left(z_{2}\right) E u_{\infty}(z) d z=0 \quad \text { for every } \varphi \in C_{0}^{1}\left(R ; \mathbb{R}^{2}\right) .
$$

Step 2: Fix a ball $B$ such that

$$
B \subset \subset\left\{s v_{1}+t v_{2}:-1<s<1,-4 L<t<-3 L\right\} .
$$

We claim that

$$
\lim _{n \rightarrow+\infty} \int_{C_{n}} \psi^{2}\left|\nabla u_{n}-\nabla u_{\infty}\right|^{2} d z=0
$$

for every $\psi \in C_{0}^{1}(R)$ vanishing in $B$. Arguing as in [12, Theorem 3.13, Step 2] we obtain that

$$
\lim _{n \rightarrow+\infty} \int_{C_{n}} \psi^{2}(z)\left(E u_{n}(z): \mathbb{C}\left(r_{n} z_{2}\right) E u_{n}(z)-E u_{\infty}(z): \mathbb{C}\left(z_{2}\right) E u_{\infty}(z)\right) d z=0,
$$

hence

$$
\lim _{n \rightarrow+\infty} \int_{C_{n}} E\left(\psi(z)\left(u_{n}(z)-u_{\infty}(z)\right)\right): \mathbb{C}_{\infty}(z) E\left(\psi(z)\left(u_{n}(z)-u_{\infty}(z)\right)\right) d z=0 .
$$

Claim (3.18) follows then from Korn's inequality (see [12, Theorem 4.2]).

Step 3: by Step 1, we deduce that $u_{\infty}$ is a weak solution of the transmission problem

$$
\begin{array}{lr}
\mu_{f} \Delta u_{\infty}^{+}+\left(\lambda_{f}+\mu_{f}\right) \nabla\left(\operatorname{div} u_{\infty}^{+}\right)=0 & \text { in } C_{\infty}^{+}, \\
\mu_{s} \Delta u_{\infty}^{-}+\left(\lambda_{s}+\mu_{s}\right) \nabla\left(\operatorname{div} u_{\infty}^{-}\right)=0 & \text { in } C_{\infty}^{-}, \\
\left(2 \mu_{f} E u_{\infty}^{+}+\lambda_{f}\left(\operatorname{div} u_{\infty}^{+}\right) I d\right) \nu_{\infty}=0 & \text { on } \Gamma_{g_{\infty}}, \\
u_{\infty}^{+}-u_{\infty}^{-}=0 & \text { on }\left\{z_{2}=0\right\}, \\
\left(2 \mu_{f} E u_{\infty}^{+}-2 \mu_{s} E u_{\infty}^{-}+\lambda_{f}\left(\operatorname{div} u_{\infty}^{+}\right) I d-\lambda_{f}\left(\operatorname{div} u_{\infty}^{-}\right) I d\right) e_{2}=0 & \text { on }\left\{z_{2}=0\right\},
\end{array}
$$

where $C_{\infty}^{+}:=C_{\infty} \cap\left\{z_{2}>0\right\}, C_{\infty}^{-}:=C_{\infty} \cap\left\{z_{2}<0\right\}, \Gamma_{g_{\infty}}:=\left\{\left(s, g_{\infty}(s)\right):-1<s<1\right\}$, $u_{\infty}^{+}:=\left.u_{\infty}\right|_{C_{\infty}^{+}}, u_{\infty}^{-}:=\left.u_{\infty}\right|_{C_{\infty}^{-}}$, and $\nu_{\infty}$ is the outer unit normal to $\Gamma_{g_{\infty}}$, wherever it exists. Note that the fourth condition in (3.19) holds because Sobolev maps are absolutely continuous on almost every line, whereas the other equations in (3.19) are a consequence of (3.17). 
In view of (4.2) and the geometry of the problem we can apply Proposition 3.5 with $R \leq 1$ to $u_{\infty}$, with $\theta_{2}=\pi, \mu_{1}=\mu_{f}, \lambda_{1}=\lambda_{f}, \mu_{2}=\mu_{s}, \lambda_{2}=\lambda_{s}$, and with data $f_{i}=0$, and

$$
g_{i}:=\left[2 \mu_{i} E u_{\infty}+\lambda_{i}\left(\operatorname{div} u_{\infty}\right) I d\right] \nu_{i} \quad \text { on } \Gamma_{i}, i=1,2,3,
$$

where $\Gamma_{1}=\partial B(O, R) \cap C_{\infty}^{+} \cap\left\{z_{1}<0\right\}, \Gamma_{2}=\partial B(O, R) \cap C_{\infty}^{-}$, and $\Gamma_{3}=\partial B(O, R) \cap C_{\infty}^{+} \cap$ $\left\{z_{1}>0\right\}$ where $O$ denotes the origin $(0,0)$. Therefore, we conclude that there exists a ball $B \subset B(O, R)$ centered in the origin, and such that

$$
u_{\infty} \in H^{3 / 2+\varepsilon}\left(B \cap\left(C_{\infty}^{+} \cup C_{\infty}^{-}\right) ; \mathbb{R}^{2}\right) .
$$

Thus, by Hölder inequality we obtain

$$
\begin{aligned}
& \int_{B(O, r) \cap C_{\infty}}\left|\nabla u_{\infty}\right|^{2} d z=\int_{B(O, r) \cap C_{\infty}^{+}}\left|\nabla u_{\infty}\right|^{2} d z+\int_{B(O, r) \cap C_{\infty}^{-}}\left|\nabla u_{\infty}\right|^{2} d z \\
& \leq r^{2-\frac{4}{s}}\left(\|\nabla u\|_{L^{s}\left(B \cap C_{\infty}^{+} ; \mathbb{M}^{2 \times 2}\right)}^{2}+\|\nabla u\|_{L^{s}\left(B \cap C_{\infty}^{-} ; \mathbb{M}^{2 \times 2}\right)}^{2}\right) \leq C r^{2 \beta}
\end{aligned}
$$

for every $r>0$ small enough, where $4<s<\frac{4}{1-2 \varepsilon}$, and $\beta=1-\frac{2}{s}>\frac{1}{2}$, where we used the fact that

$$
H^{3 / 2+\varepsilon}\left(B \cap\left(C_{\infty}^{+} \cup C_{\infty}^{-}\right) ; \mathbb{R}^{2}\right) \subset L^{s}\left(B \cap\left(C_{\infty}^{+} \cup C_{\infty}^{-}\right) ; \mathbb{R}^{2}\right)
$$

for every $s \in\left[1, \frac{4}{1-2 \varepsilon}\right]$. Choosing $\tau_{0}$ such that

$$
\tau_{0} C_{\infty} \subset\left(B(O, 1) \cap C_{\infty}\right) \backslash\left\{s e_{1}+t e_{2}:-1<s<1,-4 L<t<-3 L\right\},
$$

by Step 2 we deduce that for $0<\tau \leq \tau_{0}$ there holds

$$
\begin{aligned}
& \lim _{n \rightarrow+\infty} \frac{\int_{C\left(z_{0}, \tau r_{n}\right)}|\nabla u|^{2} d x d y}{\int_{C\left(z_{0}, r_{n}\right)}|\nabla u|^{2} d x d y}=\frac{1}{\left|C_{\infty}\right|} \lim _{n \rightarrow+\infty} \int_{\tau C_{n}}\left|\nabla u_{n}\right|^{2} d z \\
& =\frac{1}{\left|C_{\infty}\right|} \int_{\tau C_{\infty}}\left|\nabla u_{\infty}\right|^{2} d z \leq \frac{1}{\left|C_{\infty}\right|} \int_{B\left(O, \frac{\tau}{\tau_{0}}\right) \cap C_{\infty}}\left|\nabla u_{\infty}\right|^{2} d z \leq \frac{C_{2} \tau^{2 \beta}}{\left|C_{\infty}\right|} .
\end{aligned}
$$

This leads to a contradiction to (3.10) provided that $C_{1} \geq \frac{C_{2} \tau^{2 \beta}}{\left|C_{\infty}\right|}$, and thus completes the proof of (3.9) in the case $h\left(x_{0}\right)=0$. The same argument works for $h\left(x_{0}\right)>0$ by noticing that in this latter scenario after the blow-up $\mathbb{C}_{\infty}\left(h\left(x_{0}\right)+r_{n} \cdot\right) \equiv \mathbb{C}_{f}$ (see also $[12$, Theorem 3.13]).

\section{Contact-Angle conditions}

This section is devoted to the proof of Theorem 2.3. For every profile function $h$ we denote by $h_{-}^{\prime}(x)$ and $h_{+}^{\prime}(x)$, respectively, the left and right derivative of $h$ in $x \in[a, b]$, whenever they exist. In the following we denote by $\theta^{*}$ the angle

$$
\theta^{*}:=\arccos \beta,
$$

where $\beta$ is the quantity defined in (2.7). We first provide a preliminary characterization of contact-angle conditions.

Proposition 4.1. Assume that the Lamé coefficients of the film and the substrate satisfy

$$
\mu_{s} \geq \mu_{f}>0 \text { and } \mu_{s}+\lambda_{s} \geq \mu_{f}+\lambda_{f}>0 .
$$

Then, for every $\mu$-local minimizer $(u, h) \in X$ of $\mathcal{F}$ and for $z_{0}:=\left(x_{0}, 0\right) \in Z_{h} \cap \Gamma_{h}^{\text {reg }}$ the following asserts hold true: 
1. For every $x_{0} \in P_{h}$ we have that $\theta^{-}\left(x_{0}\right), \theta^{+}\left(x_{0}\right) \in\left[0, \theta^{*}\right]$ and, if $\theta^{-}\left(x_{0}\right)=\theta^{+}\left(x_{0}\right)$ then $\theta^{-}\left(x_{0}\right)=\theta^{+}\left(x_{0}\right)=0$,

2. For any $(c, d) \in I_{h}$, there holds $\theta^{-}(c), \theta^{+}(d) \in\left[0, \theta^{*}\right]$.

Additionally, $\Gamma_{h}^{j u m p}$ satisfies the following property

3. If $\beta \neq 0$, then $\Gamma_{h}^{j u m p} \cap Z_{h}=\emptyset$.

Proof. Let $(u, h)$ be a $\mu$-local minimizer of $\mathcal{F}$, and let $z_{0}=\left(x_{0}, h\left(x_{0}\right)\right) \in Z_{h} \cap\left(\Gamma_{h}^{r e g} \cup\right.$ $\left.\Gamma_{h}^{j u m p}\right)$. As a consequence of Assertion 1. of Proposition 3.3 there exist $a^{\prime}>0$ and $b^{\prime}>0$ such that the function $g:\left(-a^{\prime}, a^{\prime}\right) \rightarrow\left(-b^{\prime}, b^{\prime}\right)$ defined as

$$
g(x):=h(x)-h\left(x_{0}\right) \quad \text { for every } x \in\left(-a^{\prime}, a^{\prime}\right)
$$

satisfies one of the following conditions:

$\left(c_{1}\right) g$ is a Lipschitz function in $\left(-a^{\prime}, a^{\prime}\right)$ with Lipschitz constant Lip $g \leq L$ for some $L>1$

$\left(c_{2}\right) g$ is a Lipschitz function in $\left(-a^{\prime}, 0\right)$ with Lipschitz constant Lip $g \leq L$ for some $L>1$, and $g_{+}^{\prime}(0)=\infty$;

$\left(c_{3}\right) g$ is a Lipschitz function in $\left(0, a^{\prime}\right)$ with Lipschitz constant Lip $g \leq L$ for some $L>1$, and $g_{-}^{\prime}(0)=-\infty$.

We also point out that in view of the internal-ball condition (see Proposition 3.2), under condition $\left(c_{1}\right)$, the angle between $g_{-}^{\prime}(0)$ and $g_{+}^{\prime}(0)$ intersecting $\Omega_{h}^{-}$is always in the interval $[\pi, 2 \pi)$.

In the following we denote the intersection of a given a set $U$ with the half-planes $\{x<0\}$ and $\{x>0\}$ by $U^{2}:=U \cap\{x<0\}$ and $U^{3}:=U \cap\{x>0\}$, respectively. We also set $U^{1}:=U$.

Choose an infinitesimal sequence $r_{n} \rightarrow 0$, and consider the sets

$$
C\left(z_{0}, r_{n}\right):=\left\{z_{0}+(x, y) \in \mathbb{R}^{2}:-r_{n}<x<r_{n},-4 L r_{n}<y<g(x)\right\},
$$

and

$$
C_{n}:=\frac{1}{r_{n}}\left(C\left(z_{0}, r_{n}\right)-z_{0}\right)=\left\{z \in \mathbb{R}^{2}:-1<z_{1}<1,-4 L<z_{2}<\frac{g\left(r_{n} z_{1}\right)}{r_{n}}\right\} .
$$

We observe that for $k=1,2,3$ we have that $\chi_{C_{n}^{k}} \rightarrow \chi_{C_{\infty}^{k}}$ a.e., where

$$
C_{\infty}:=\left\{z \in \mathbb{R}^{2}:-1<z_{1}<1,-4 L<z_{2}<g_{\infty}\left(z_{1}\right)\right\},
$$

the function $g_{\infty}:(-1,1) \rightarrow \mathbb{R}$ is defined as

$$
g_{\infty}\left(z_{1}\right):= \begin{cases}g_{-}^{\prime}(0) z_{1} & \text { for } z_{1}<0, \\ g_{+}^{\prime}(0) z_{1} & \text { for } z_{1}>0 .\end{cases}
$$

and $\chi_{C_{n}^{k}}, \chi_{C_{\infty}^{k}}$ denote the characteristic functions of the sets $C_{n}^{k}$ and $C_{\infty}^{k}$, respectively. In particular, $C_{\infty}^{k} \subset R^{k}$ for $k=1,2,3$, where

$$
R:=\left\{z \in \mathbb{R}^{2}:-1<z_{1}<1,-4 L<z_{2}<4 L\right\} .
$$

With a slight abuse of notation under each condition $\left(c_{k}\right)$ we identify the map $u$ with its $H^{1}$-extension to the set $\Omega_{h} \cup R^{k}\left(z_{0}, r_{n}\right)$, where

$$
R\left(z_{0}, r_{n}\right):=z_{0}+r_{n} R .
$$

Note that this extension is well-defined owing to Assertion 1. of Proposition 3.3, which guarantees that the graph of $h$, aside from cusps and cuts, is locally Lipschitz. 
Let $I^{k}$ be defined as

$$
I^{k}:= \begin{cases}(-1,1) & \text { if } k=1 \\ (-1,0) & \text { if } k=2 \\ (0,1) & \text { if } k=3\end{cases}
$$

For every $0<\delta<1$ under each condition $\left(c_{k}\right)$ we consider a function $\psi_{\delta} \in W^{1, \infty}\left(I^{k}\right)$ to be specified later, satisfying the following properties

$$
\begin{aligned}
& \sup _{\delta}\left\|\psi_{\delta}\right\|_{L^{\infty}\left(I^{k}\right)} \leq C, \\
& 0 \leq g_{\infty}\left(z_{1}\right)+\delta \psi_{\delta}\left(z_{1}\right)<4 L \quad \text { for every } z_{1} \in I^{k}, \\
& \operatorname{supp} \psi_{\delta}=\left[x_{\delta}^{-}, x_{\delta}^{+}\right],
\end{aligned}
$$

with

$$
\begin{cases}x_{\delta}^{-}<0<x_{\delta}^{+} & \text {for condition }\left(c_{1}\right), \\ x_{\delta}^{-}<0 \text { and } x_{\delta}^{+}=0 & \text { for condition }\left(c_{2}\right), \\ x_{\delta}^{-}=0 \text { and } x_{\delta}^{+}>0 & \text { for condition }\left(c_{3}\right)\end{cases}
$$

Define the maps

$$
\psi_{\delta}^{n}(x):= \begin{cases}r_{n} \psi_{\delta}\left(\frac{x-x_{0}}{r_{n}}\right) & \text { for every } x \in\left[x_{0}+r_{n} x_{\delta}^{-}, x_{0}+r_{n} x_{\delta}^{+}\right] \\ 0 & \text { otherwise in }(a, b) .\end{cases}
$$

Note that for $r_{n}$ small enough,

$$
\Omega_{h+\delta \psi_{\delta}^{n}} \subset \Omega_{h} \cup R^{k}\left(z_{0}, r_{n}\right),
$$

and

$$
\left|\Omega_{h+\delta \psi_{\delta}^{n}} \Delta \Omega_{h}\right| \leq \frac{\mu}{2} .
$$

By Proposition 3.1 there exists $\lambda_{0}>0$ such that

$$
\mathcal{F}(u, h)=\min \left\{\mathcal{F}(v, \tilde{h})+\lambda|| \Omega_{h}^{+}|-| \Omega_{\tilde{h}}^{+}||:(v, \tilde{h}) \in X,\left|\Omega_{\tilde{h}} \Delta \Omega_{h}\right| \leq \frac{\mu}{2}\right\}
$$

for all $\lambda \geq \lambda_{0}$. In the following we denote by $\mathcal{G}$ the volume-penalized functional defined as

$$
\mathcal{G}(v, \tilde{h}):=\mathcal{F}(v, \tilde{h})+\lambda_{0}|| \Omega_{h}^{+}|-| \Omega_{\tilde{h}}^{+}||
$$

for every $(v, \tilde{h}) \in X$. By the minimality of $(u, h)$, and by (1.2), (2.4), (2.7), (4.7), and (4.8), there holds

$$
0 \leq \frac{\mathcal{G}\left(u, h+\delta \psi_{\delta}^{n}\right)-\mathcal{G}(u, h)}{\delta r_{n}}:=A_{n}^{1}-A_{n}^{2}+B_{n}+D_{n}+E_{n},
$$

where

$$
\begin{aligned}
A_{n}^{1} & :=\frac{1}{\delta r_{n}} \int_{\Omega_{h+\delta \psi_{n} \backslash \Omega_{h}}} W_{0}\left(y, E u(x, y)-E_{0}(y)\right) d x d y, \\
A_{n}^{2} & :=\frac{1}{\delta r_{n}} \int_{\Omega_{h} \backslash \Omega_{h+\delta \psi_{\delta}^{n}}} W_{0}\left(y, E u(x, y)-E_{0}(y)\right) d x d y, \\
B_{n} & :=\frac{\gamma_{f} \beta}{\delta r_{n}}\left(\mathcal{H}^{1}\left(\tilde{\Gamma}_{h+\delta \psi_{\delta}^{n}} \cap\{y=0\}\right)-\mathcal{H}^{1}\left(\tilde{\Gamma}_{h} \cap\{y=0\}\right)\right) \\
+ & \frac{\gamma_{f}}{\delta r_{n}}\left(\mathcal{H}^{1}\left(\tilde{\Gamma}_{h+\delta \psi_{\delta}^{n}} \cap\{y>0\}\right)-\mathcal{H}^{1}\left(\tilde{\Gamma}_{h} \cap\{y>0\}\right)\right),
\end{aligned}
$$




$$
D_{n}:=\frac{\lambda_{0}}{\delta r_{n}}\left|\int_{a}^{b} h-\left(h+\delta \psi_{\delta}^{n}\right) d x\right|,
$$

and

$$
E_{n}:=\frac{2 \gamma_{f}}{\delta r_{n}} \sum_{x \in C(h)}\left(\left(h+\delta \psi_{\delta}^{n}\right)^{-}(x)-\left(h+\delta \psi_{\delta}^{n}\right)(x)\right)-\frac{2 \gamma_{f}}{\delta r_{n}} \sum_{x \in C(h)}\left(h^{-}(x)-h(x)\right) .
$$

We begin by noticing that

$$
E_{n}=0
$$

by the regularity of $\psi_{\delta}^{n}$, and that

$$
D_{n} \leq \frac{\lambda_{0}}{r_{n}} \int_{r_{n} x_{\delta}^{-}+x_{0}}^{r_{n} x_{\delta}^{+}+x_{0}}\left|\psi_{\delta}^{n}(x)\right| d x=\lambda_{0} r_{n} \int_{x_{\delta}^{-}}^{x_{\delta}^{+}}\left|\psi_{\delta}\left(z_{1}\right)\right| d z_{1} \rightarrow 0
$$

as $n \rightarrow+\infty$, by the change of variable

$$
x=x_{0}+r_{n} z_{1} .
$$

Step 1 (Convergence of the elastic-energy terms). We show that $A_{n}^{1} \rightarrow 0$. The proof that $A_{n}^{2} \rightarrow 0$ is analogous. We begin by assuming that the quantities

$$
\lambda_{n}:=\frac{1}{r_{n}}\left(\int_{C\left(z_{0}, r_{n}\right)}|\nabla u|^{2} d x d y\right)^{\frac{1}{2}}
$$

satisfy

$$
\limsup _{n \rightarrow+\infty} \lambda_{n}<+\infty
$$

In this situation we define the maps $v_{n}: C_{n}^{k} \rightarrow \mathbb{R}^{2}$, as

$$
v_{n}(z):=\frac{u\left(z_{0}+r_{n} z\right)-f_{C^{k}\left(z_{0}, r_{n}\right)} u(x, y) d x d y}{r_{n}} .
$$

Notice that by construction we have $\int_{C_{n}^{k}} v_{n}(x, y) d x d y=0$. Since $u \in H^{1}\left(\Omega_{h} \cup\right.$ $\left.R\left(z_{0}, r_{n}\right)^{k} ; \mathbb{R}^{2}\right)$, in each case $\left(c_{k}\right), k=1,2,3$, the map $v_{n}$ satisfies $v_{n} \in H^{1}\left(R^{k} ; \mathbb{R}^{2}\right)$, $k=1,2,3$, and

$$
\left\|v_{n}\right\|_{W^{1,2}\left(R^{k} ; \mathbb{R}^{2}\right)} \leq C\left\|\nabla v_{n}\right\|_{L^{2}\left(C_{n}^{k} ; \mathbb{M}^{2 \times 2}\right)} \leq C \lambda_{n}^{2} \leq C
$$

for $n$ big enough, where the last inequality follows from (4.18). Therefore for each case $\left(c_{k}\right), k=1,2,3$ we conclude that

$$
\begin{aligned}
A_{n}^{1} & =\frac{1}{\delta r_{n}} \int_{I_{n}^{\delta}} \int_{h(x)}^{h(x)+\delta \psi_{\delta}^{n}(x)} \mathbb{C}_{f}\left(E u(x, y)-E_{0}(y)\right):\left(E u(x, y)-E_{0}(y)\right) d y d x \\
& =\frac{r_{n}}{\delta} \int_{I^{\delta}} \int_{\frac{h\left(x_{0}+r_{n} z_{1}\right)}{r_{n}}}^{\frac{h\left(x_{0}+r_{n} z_{1}\right)}{r_{n}}+\delta \psi_{\delta}\left(z_{1}\right)} \mathbb{C}_{f}\left(E v_{n}(z)-E_{0}\left(z_{2}\right)\right):\left(E v_{n}(z)-E_{0}\left(z_{2}\right)\right) d z_{2} d z_{1} \\
& \leq \frac{C r_{n}}{\delta}\left\|E v_{n}-E_{0}\right\|_{L^{2}\left(R^{k} ; \mathbb{M}_{\mathrm{sym}}^{2 \times 2}\right)} \leq \frac{C r_{n}}{\delta},
\end{aligned}
$$

with $I_{n}^{\delta}:=\left(x_{0}+r_{n} x_{\delta}^{-}, x_{0}+r_{n} x_{\delta}^{+}\right) \cap\left\{\psi_{\delta}^{n} \geq 0\right\}, I^{\delta}:=\left(x_{\delta}^{-}, x_{\delta}^{+}\right) \cap\left\{\psi_{\delta} \geq 0\right\}$, where in the second equality we performed the change of variable

$$
(x, y)=\left(x_{0}+r_{n} z_{1}, r_{n} z_{2}\right)
$$

and where the last inequality follows from (4.19), and (4.3)-(4.6). 
When (4.18) does not hold, we have

$$
\limsup _{n \rightarrow+\infty} \lambda_{n}=+\infty
$$

Then, in view of Proposition 3.6 there holds

$$
r_{n} \lambda_{n}^{2}=\frac{1}{r_{n}} \int_{C\left(z_{0}, r_{n}\right)}|\nabla u|^{2} d x d y \leq C r_{n}^{2 \alpha-1} \rightarrow 0 .
$$

We define the maps $w_{n}: C_{n}^{k} \rightarrow \mathbb{R}^{2}$, as

$$
w_{n}(z):=\frac{u\left(z_{0}+r_{n} z\right)-f_{C^{k}\left(z_{0}, r_{n}\right)} u(x, y) d x d y}{\lambda_{n} r_{n}},
$$

for every $z \in C_{n}^{k}$. Note that $\int_{C_{n}^{k}} w_{n}(x) d x=0$ by construction. Again, the fact that $u \in H^{1}\left(\Omega_{h} \cup R^{k}\left(z_{0}, r_{n}\right) ; \mathbb{R}^{2}\right)$ implies that $w_{n} \in H^{1}\left(R^{k} ; \mathbb{R}^{2}\right)$, with

$$
\left\|w_{n}\right\|_{W^{1,2}\left(R^{k} ; \mathbb{R}^{2}\right)} \leq C\left\|\nabla w_{n}\right\|_{L^{2}\left(C_{n}^{k} ; \mathbb{M}^{2 \times 2}\right)} \leq C .
$$

By employing the same change of variable (4.20) of the first case we observe that

$$
\begin{aligned}
A_{n}^{1} & =\frac{1}{\delta r_{n}} \int_{I_{n}^{\delta}} \int_{h(x)}^{h(x)+\delta \psi_{\delta}^{n}(x)} \mathbb{C}_{f}\left(E u(x, y)-E_{0}(y)\right):\left(E u(x, y)-E_{0}(y)\right) d y d x \\
& =\frac{\lambda_{n}^{2} r_{n}}{\delta} \int_{I^{\delta}} \int_{\frac{h\left(x_{0}+r_{n} z_{1}\right)}{r_{n}}}^{\frac{h\left(x_{0}+r_{n} z_{1}\right)}{r_{n}}+\delta \psi_{\delta}\left(z_{1}\right)} \mathbb{C}_{f}\left(E w_{n}(z)-E_{0}\left(z_{2}\right)\right):\left(E w_{n}(z)-E_{0}\left(z_{2}\right)\right) d z_{2} d z_{1} \\
& \leq \frac{C \lambda_{n}^{2} r_{n}}{\delta}\left\|E w_{n}-E_{0}\right\|_{L^{2}\left(R^{k} ; \mathbb{M}_{\mathrm{sym}}^{2 \times 2}\right)} \leq \frac{C}{\delta} \lambda_{n}^{2} r_{n}
\end{aligned}
$$

where now in the last inequality we used (4.22) and (4.3)-(4.6). The claim follows from (4.21).

Step 2 (Surface-energy convergence under condition $\left(c_{1}\right)$ ). In this step we study the convergence of the terms $B_{n}$ under condition $\left(c_{1}\right)$. To this aim, we treat in three different subsections the cases of island borders, of valleys with no vanishing contact angles, and of valleys with one vanishing contact angle. In particular, the first subsection yields Assertion 2. of the proposition, whereas Assertion 1. is proved in the second and third subsections.

Island borders. In this subsection we prove Assertion 2. of the proposition, namely we consider $x_{0}=c$ for some $(c, d) \in I_{h}$, and we prove that $\theta^{-}(c) \leq \theta^{*}$ (see Figure 8). The case of $x_{0}=d$ and $\theta^{+}(d)$ is analogous by symmetry. For simplicity we denote in the following $\theta^{-}(c)$ by $\theta$.

We begin by considering $\theta^{*}>0$. Note that

$$
\tan (\theta)=-g_{-}^{\prime}(0) \text { and } \tan \left(\theta^{*}\right)=\frac{\sqrt{1-\beta^{2}}}{\beta} .
$$

Assume by contradiction that

$$
\theta>\theta^{*}
$$


Then by (4.23), we have $0<\tan \left(\theta^{*}\right)<-g_{-}^{\prime}(0)$. We define $\psi_{\delta}$ by

$$
\psi_{\delta}(s)= \begin{cases}-\left(\frac{g_{-}^{\prime}(0)+\tan \left(\theta^{*}\right)}{\delta}\right) s+\frac{\tan \left(\theta^{*}\right)}{g_{-}^{\prime}(0)}+1 & \text { for } \frac{\delta}{g_{-}^{\prime}(0)}<s \leq 0, \\ \frac{-\tan \left(\theta^{*}\right)}{\delta} s+\frac{\tan \left(\theta^{*}\right)}{g_{-}^{\prime}(0)}+1 & \text { for } 0<s<\delta\left(\frac{1}{\tan \left(\theta^{*}\right)}+\frac{1}{g_{-}^{\prime}(0)}\right), \\ 0 & \text { otherwise. }\end{cases}
$$

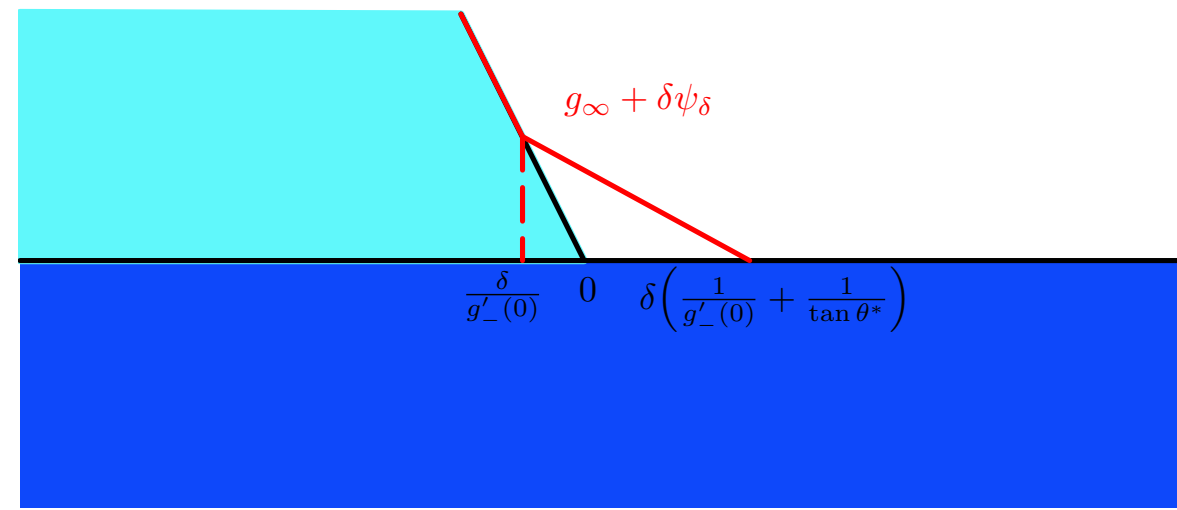

Figure 8. The blow-up at island borders (and at valleys with one vanishing contact angle) is displayed. The profile of $g_{\infty}$ and the perturbation $g_{\infty}+\delta \psi_{\delta}$ in the case $\theta \geq \theta^{*}=\arccos \beta$ for island borders are highlighted in black and in red, respectively

We observe that

$$
\mathcal{H}^{1}\left(\tilde{\Gamma}_{h+\delta \psi_{\delta}^{n}} \cap\{y=0\}\right)-\mathcal{H}^{1}\left(\tilde{\Gamma}_{h} \cap\{y=0\}\right)=-\delta r_{n}\left(\frac{1}{\tan \left(\theta^{*}\right)}+\frac{1}{g_{-}^{\prime}(0)}\right)
$$

as shown in Figure 8. We now observe that by condition $\left(c_{1}\right)$ the map $h$ is Lipschitz in $\left(-a^{\prime}, a^{\prime}\right)$ and hence, its derivative $h^{\prime}$ exists a.e. in $\left(-a^{\prime}, a^{\prime}\right)$, and $h_{-}^{\prime}$ and $h_{+}^{\prime}$ are, respectively, left and right continuous. These properties together with the definition of $\psi_{\delta}$ imply that

$$
\begin{aligned}
B_{n} & =\frac{\gamma_{f}}{\delta r_{n}} \int_{\frac{\delta r_{n}}{g_{-}^{\prime}(0)}+x_{0}}^{\delta r_{n}\left(\frac{1}{\tan \left(\theta^{*}\right)}+\frac{1}{g_{-}^{\prime}(0)}\right)+x_{0}} \sqrt{1+\left(h^{\prime}(x)+\delta \psi_{\delta}^{\prime}\left(\frac{x-x_{0}}{r_{n}}\right)\right)^{2}} d x \\
& -\frac{\gamma_{f}}{\delta r_{n}} \int_{\frac{\delta r_{n}}{g_{-}^{\prime}(0)}+x_{0}}^{x_{0}} \sqrt{1+\left(h^{\prime}(x)\right)^{2}} d x-\gamma_{f} \beta\left(\frac{1}{\tan \left(\theta^{*}\right)}+\frac{1}{g_{-}^{\prime}(0)}\right) \\
& =\frac{\gamma_{f}}{\delta} \int_{\frac{\delta}{g_{-}^{\prime}(0)}}^{\delta\left(\frac{1}{\tan \left(\theta^{*}\right)}+\frac{1}{g_{-}^{\prime}(0)}\right)} \sqrt{1+\left(h^{\prime}\left(x_{0}+r_{n} s\right)+\delta \psi_{\delta}^{\prime}(s)\right)^{2}} d s \\
& -\frac{\gamma_{f}}{\delta} \int_{\frac{\delta}{g_{-}^{\prime}(0)}}^{0} \sqrt{1+\left(h^{\prime}\left(x_{0}+r_{n} s\right)\right)^{2}} d s-\gamma_{f} \beta\left(\frac{1}{\tan \left(\theta^{*}\right)}+\frac{1}{g_{-}^{\prime}(0)}\right),
\end{aligned}
$$


where in the last equality we used the change of variable (4.17). Furthermore, in view of the fact that $h_{-}^{\prime}\left(x_{0}+r_{n} z\right) \rightarrow g_{-}^{\prime}(0)$ and $h_{+}^{\prime}\left(x_{0}+r_{n} z\right) \rightarrow g_{+}^{\prime}(0)$ as $n \rightarrow+\infty$, the Lebesgue Dominated Convergence Theorem yields that

$$
B_{n} \rightarrow-\gamma_{f} \beta\left(\frac{1}{\tan \left(\theta^{*}\right)}+\frac{1}{g_{-}^{\prime}(0)}\right)+\frac{\gamma_{f}}{\beta \tan \left(\theta^{*}\right)}+\gamma_{f} \frac{\sqrt{1+\left(g_{-}^{\prime}(0)\right)^{2}}}{g_{-}^{\prime}(0)} .
$$

By (4.9), (4.16), and Step 1, there holds

$$
-\beta\left(\frac{1}{\tan \left(\theta^{*}\right)}+\frac{1}{g_{-}^{\prime}(0)}\right)+\frac{1}{\beta \tan \left(\theta^{*}\right)}+\frac{\sqrt{1+\left(g_{-}^{\prime}(0)\right)^{2}}}{g_{-}^{\prime}(0)} \geq 0
$$

which in turn implies

$$
\beta \tan \left(\theta^{*}\right) \sqrt{1+(\tan (\theta))^{2}} \leq\left(1-\beta^{2}\right) \tan (\theta)+\beta^{2} \tan \left(\theta^{*}\right) .
$$

Substituting (4.23) in (4.27), dividing by $\sqrt{1-\beta^{2}}$, and taking the squares of both sides of the resulting inequality, we obtain

$$
\left(\beta \tan (\theta)-\sqrt{1-\beta^{2}}\right)^{2} \leq 0
$$

and hence, again by (4.23), $\theta=\theta^{*}$ which is in contradiction with (4.24).

Consider now the case in which $\theta^{*}=0$, i.e., $\beta=1$. Assume by contradiction that

$$
\theta>\theta^{*}=0 \text {. }
$$

Then, for $\delta$ small enough, by (4.23), we have $0=\tan \left(\theta^{*}\right)<\delta<-g_{-}^{\prime}(0)$. We define $\psi_{\delta}$ by

$$
\psi_{\delta}(s)= \begin{cases}-\left(\frac{g_{-}^{\prime}(0)+\varepsilon_{\delta}}{\delta}\right) s+\frac{\varepsilon_{\delta}}{g_{-}^{\prime}(0)}+1 & \text { for } \frac{\delta}{g_{-}^{\prime}(0)}<s \leq 0 \\ \frac{-\varepsilon_{\delta}}{\delta} s+\frac{\varepsilon_{\delta}}{g_{-}^{\prime}(0)}+1 & \text { for } 0<s<\delta\left(\frac{1}{\varepsilon_{\delta}}+\frac{1}{g_{-}^{\prime}(0)}\right) \\ 0 & \text { otherwise }\end{cases}
$$

where $\varepsilon_{\delta}<<\delta$ is such that $\delta\left(\frac{1}{\varepsilon_{\delta}}+\frac{1}{g_{-}^{\prime}(0)}\right)<1$.

The same computations as in the case $\theta^{*}>0$ yield

$$
\mathcal{H}^{1}\left(\tilde{\Gamma}_{h+\delta \psi_{\delta}^{n}} \cap\{y=0\}\right)-\mathcal{H}^{1}\left(\tilde{\Gamma}_{h} \cap\{y=0\}\right)=-\delta r_{n}\left(\frac{1}{\varepsilon_{\delta}}+\frac{1}{g_{-}^{\prime}(0)}\right),
$$

and hence, since here $\beta=1$,

$$
\begin{aligned}
& B_{n}=\frac{\gamma_{f}}{\delta r_{n}} \int_{\frac{\delta r_{n}}{g_{-}^{\prime}(0)}+x_{0}}^{\delta r_{n}\left(\frac{1}{\varepsilon_{\delta}}+\frac{1}{g_{-}^{\prime}(0)}\right)+x_{0}} \sqrt{1+\left(h^{\prime}(x)+\delta \psi_{\delta}^{\prime}\left(\frac{x-x_{0}}{r_{n}}\right)\right)^{2}} d x \\
& -\frac{\gamma_{f}}{\delta r_{n}} \int_{\frac{\delta r_{n}}{g_{-}^{\prime}(0)}+x_{0}}^{x_{0}} \sqrt{1+\left(h^{\prime}(x)\right)^{2}} d x-\gamma_{f}\left(\frac{1}{\varepsilon_{\delta}}+\frac{1}{g_{-}^{\prime}(0)}\right) \\
& =\frac{\gamma_{f}}{\delta} \int_{\frac{\delta}{g_{-}^{\prime}(0)}}^{\delta\left(\frac{1}{\varepsilon_{\delta}}+\frac{1}{g_{-}^{\prime}(0)}\right)} \sqrt{1+\left(h^{\prime}\left(x_{0}+r_{n} s\right)+\delta \psi_{\delta}^{\prime}(s)\right)^{2}} d s \\
& -\frac{\gamma_{f}}{\delta} \int_{\frac{\delta}{g_{-}^{\prime}(0)}}^{0} \sqrt{1+\left(h^{\prime}\left(x_{0}+r_{n} s\right)\right)^{2}} d s-\gamma_{f}\left(\frac{1}{\varepsilon_{\delta}}+\frac{1}{g_{-}^{\prime}(0)}\right),
\end{aligned}
$$


which in turn, by the Dominated Convergence Theorem, implies

$$
B_{n} \rightarrow-\gamma_{f}\left(\frac{1}{\varepsilon_{\delta}}+\frac{1}{g_{-}^{\prime}(0)}\right)+\gamma_{f} \frac{\sqrt{1+\varepsilon_{\delta}^{2}}}{\varepsilon_{\delta}}+\gamma_{f} \frac{\sqrt{1+\left(g_{-}^{\prime}(0)\right)^{2}}}{g_{-}^{\prime}(0)} .
$$

Since the function $x \rightarrow \frac{\sqrt{1+x^{2}}}{x}-\frac{1}{x}$ is strictly increasing in $(-\infty, 0)$, inequality (4.30) gives

$$
0>g_{-}^{\prime}(0) \geq-\varepsilon_{\delta}
$$

By the arbitrary smallness of $\varepsilon_{\delta}$ we conclude that $g_{-}^{\prime}(0)=0$. This contradicts (4.29), and completes the proof of Assertion 2. of the proposition.

Valleys with no vanishing contact angles. In this subsection we begin the proof of Assertion 1. of the proposition, namely we consider a point $x_{0} \in P_{h}$ and we prove that at least one between $g_{-}^{\prime}(0)$ and $g_{+}^{\prime}(0)$ is zero. Notice that since the profile of the film is a graph we have

Assume by contradiction that

$$
g_{-}^{\prime}(0) \leq 0 \leq g_{+}^{\prime}(0) .
$$

$$
g_{-}^{\prime}(0)<0<g_{+}^{\prime}(0),
$$

and define $\psi_{\delta}$ by

$$
\psi_{\delta}(s):= \begin{cases}0 & \text { for } s<\frac{\delta}{g_{-}^{\prime}(0)} \text { and } s>\frac{\delta}{g_{+}^{\prime}(0)} . \\ \frac{\delta-g_{\infty}(s)}{\delta} & \text { for } s \in\left[\frac{\delta}{g_{-}^{\prime}(0)}, \frac{\delta}{g_{+}^{\prime}(0)}\right]\end{cases}
$$

for every $s \in(-1,1)$ (see Figure 9 ). Since $\psi_{\delta} \geq 0$, by (4.12) we obtain

$$
B_{n}=\frac{\gamma_{f}}{\delta r_{n}}\left(\mathcal{H}\left(\tilde{\Gamma}_{h+\delta \psi_{\delta}^{n}} \cap\{y>0\}\right)-\mathcal{H}\left(\tilde{\Gamma}_{h} \cap\{y>0\}\right)\right) .
$$

As before, since $h$ is Lipschitz in $\left(-a^{\prime}, a^{\prime}\right)$, the definition of $\psi_{\delta}$ implies that

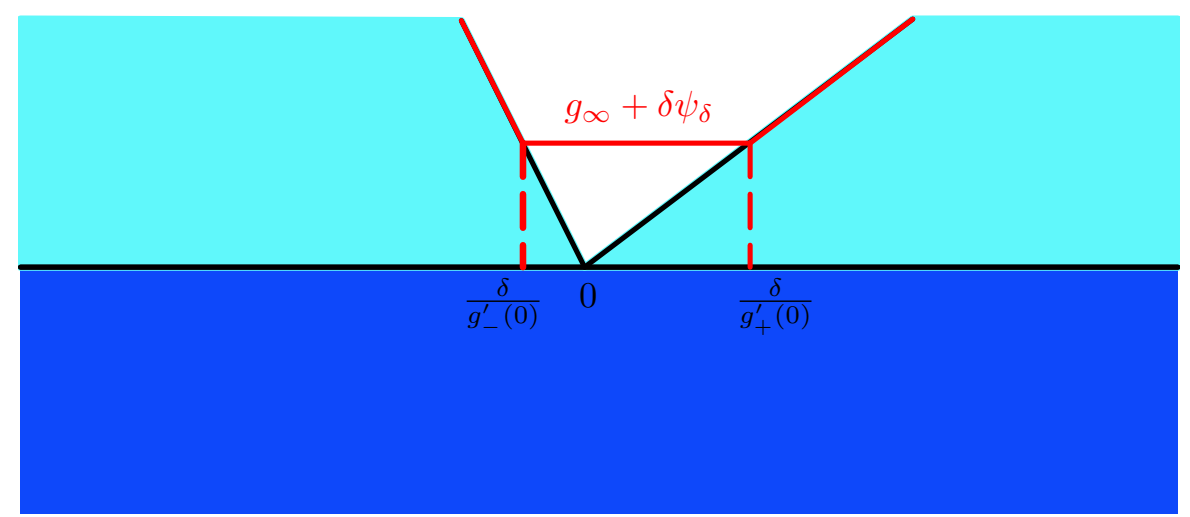

Figure 9. The blow-up at a valley with no vanishing contact angle is displayed. The profile of $g_{\infty}$ and the perturbation $g_{\infty}+\delta \psi_{\delta}$ are highlighted in black and in red, respectively

$$
B_{n}=\frac{\gamma_{f}}{\delta r_{n}} \int_{\frac{\delta r_{n}}{g_{-}^{\prime}(0)}+x_{0}}^{\frac{\delta r_{n}}{g_{+}^{\prime}(0)}+x_{0}}\left(\sqrt{1+\left(h^{\prime}(x)+\delta \psi_{\delta}^{\prime}\left(\frac{x-x_{0}}{r_{n}}\right)\right)^{2}}-\sqrt{1+\left(h^{\prime}(x)\right)^{2}}\right) d x
$$




$$
\begin{aligned}
& =\frac{\gamma_{f}}{\delta r_{n}} \int_{\frac{\delta r_{n}}{g_{-}^{\prime}(0)}+x_{0}}^{x_{0}}\left(\sqrt{1+\left(h^{\prime}(x)-g_{-}^{\prime}(0)\right)^{2}}-\sqrt{1+\left(h^{\prime}(x)\right)^{2}}\right) d x \\
& +\frac{\gamma_{f}}{\delta r_{n}} \int_{x_{0}}^{\frac{\delta r_{n}}{g_{+}^{\prime}(0)}+x_{0}}\left(\sqrt{1+\left(h^{\prime}(x)-g_{+}^{\prime}(0)\right)^{2}}-\sqrt{1+\left(h^{\prime}(x)\right)^{2}}\right) d x \\
& =\frac{\gamma_{f}}{\delta} \int_{\frac{\delta}{g_{-}^{\prime}(0)}}^{0}\left(\sqrt{1+\left(h_{-}^{\prime}\left(x_{0}+r_{n} z\right)-g_{-}^{\prime}(0)\right)^{2}}-\sqrt{1+\left(h_{-}^{\prime}\left(x_{0}+r_{n} z\right)\right)^{2}}\right) d z \\
& +\frac{\gamma_{f}}{\delta} \int_{0}^{\frac{\delta}{g_{+}^{\prime}(0)}}\left(\sqrt{1+\left(h_{+}^{\prime}\left(x_{0}+r_{n} z\right)-g_{+}^{\prime}(0)\right)^{2}}-\sqrt{1+\left(h_{+}^{\prime}\left(x_{0}+r_{n} z\right)\right)^{2}}\right) d x
\end{aligned}
$$

where in the last equality we used the change of variable (4.17). Furthermore, in view of the fact that $h_{-}^{\prime}\left(x_{0}+r_{n} z\right) \rightarrow g_{-}^{\prime}(0)$ and $h_{+}^{\prime}\left(x_{0}+r_{n} z\right) \rightarrow g_{+}^{\prime}(0)$ as $n \rightarrow+\infty$, the Lebesgue Dominated Convergence Theorem yields that

$$
B_{n} \rightarrow \gamma_{f}\left(\frac{g_{-}^{\prime}(0)}{1+\sqrt{1+\left(g_{-}^{\prime}(0)\right)^{2}}}-\frac{g_{+}^{\prime}(0)}{1+\sqrt{1+\left(g_{+}^{\prime}(0)\right)^{2}}}\right) .
$$

as $n \rightarrow+\infty$. By (4.9), (4.15), (4.16), and Step 1, there holds

$$
\frac{g_{-}^{\prime}(0)}{1+\sqrt{1+\left(g_{-}^{\prime}(0)\right)^{2}}} \geq \frac{g_{+}^{\prime}(0)}{1+\sqrt{1+\left(g_{+}^{\prime}(0)\right)^{2}}} .
$$

We observe that, setting $f(x):=\frac{x}{1+\sqrt{1+x^{2}}}$ for every $x \in \mathbb{R}$, there holds $f^{\prime}(x)>0$ for every $x \in \mathbb{R}$. Thus (4.32) yields that $g_{-}^{\prime}(0) \geq g_{+}^{\prime}(0)$ which is in contradiction with (4.31).

Valleys with one vanishing contact angle. In this subsection we conclude the proof of Assertion 1. of the proposition. From the previous subsection it remains to prove that if $x_{0} \in P_{h}$ is such that $\theta^{+}\left(x_{0}\right)=0$, then $\theta^{-}\left(x_{0}\right) \leq \theta^{*}$ (see Figure 8). In the symmetric case, in which $x_{0} \in P_{h}$ is such that $\theta^{-}\left(x_{0}\right)=0$, analogous arguments imply that $\theta^{+}\left(x_{0}\right) \leq \theta^{*}$.

Let $x_{0} \in P_{h}$ with $\theta^{+}\left(x_{0}\right)=0$. We first consider the case $\theta^{*}:=\arccos (\beta)>0$. Assume by contradiction that

$$
\theta:=\theta^{-}\left(x_{0}\right)>\theta^{*} .
$$

We define $\psi_{\delta}$ as in the case of island borders, by

$$
\psi_{\delta}(s)= \begin{cases}-\left(\frac{g_{-}^{\prime}(0)+\tan \left(\theta^{*}\right)}{\delta}\right) s+\frac{\tan \left(\theta^{*}\right)}{g_{-}^{\prime}(0)}+1 & \text { for } \frac{\delta}{g_{-}^{\prime}(0)}<s \leq 0, \\ \frac{-\tan \left(\theta^{*}\right)}{\delta} s+\frac{\tan \left(\theta^{*}\right)}{g_{-}^{\prime}(0)}+1 & \text { for } 0<s<\delta\left(\frac{1}{\tan \left(\theta^{*}\right)}+\frac{1}{g_{-}^{\prime}(0)}\right), \\ 0 & \text { otherwise. }\end{cases}
$$

Differently from the case of island borders, we have

$$
\mathcal{H}^{1}\left(\tilde{\Gamma}_{h+\delta \psi_{\delta}^{n}} \cap\{y=0\}\right)-\mathcal{H}^{1}\left(\tilde{\Gamma}_{h} \cap\{y=0\}\right)=0,
$$

and

$$
\begin{aligned}
B_{n} & =\frac{\gamma_{f}}{\delta r_{n}} \int_{\frac{\delta r_{n}}{g_{-}^{\prime}(0)}+x_{0}}^{\delta r_{n}\left(\frac{1}{\tan \left(\theta^{*}\right)}+\frac{1}{g_{-}^{\prime}(0)}\right)+x_{0}} \sqrt{1+\left(h^{\prime}(x)+\delta \psi_{\delta}^{\prime}\left(\frac{x-x_{0}}{r_{n}}\right)\right)^{2}} d x \\
& -\frac{\gamma_{f}}{\delta r_{n}} \int_{\frac{\delta r_{n}}{g_{-}^{\prime}(0)}+x_{0}}^{x_{0}} \sqrt{1+\left(h^{\prime}(x)\right)^{2}} d x-\gamma_{f}\left(\frac{1}{\tan \left(\theta^{*}\right)}+\frac{1}{g_{-}^{\prime}(0)}\right)
\end{aligned}
$$




$$
\begin{aligned}
& =\frac{\gamma_{f}}{\delta} \int_{\frac{\delta}{g_{-}^{\prime}(0)}}^{\delta}\left(\frac{1}{\tan \left(\theta^{*}\right)}+\frac{1}{g_{-}^{\prime}(0)}\right) \sqrt{1+\left(h^{\prime}\left(x_{0}+r_{n} s\right)+\delta \psi_{\delta}^{\prime}(s)\right)^{2}} d s \\
& -\frac{\gamma_{f}}{\delta} \int_{\frac{\delta}{g_{-}^{\prime}(0)}}^{0} \sqrt{1+\left(h^{\prime}\left(x_{0}+r_{n} s\right)\right)^{2}} d s-\gamma_{f}\left(\frac{1}{\tan \left(\theta^{*}\right)}+\frac{1}{g_{-}^{\prime}(0)}\right) .
\end{aligned}
$$

Arguing as in Step 2 in the case of island borders, by the Dominated Convergence Theorem, we obtain

$$
B_{n} \rightarrow-\gamma_{f}\left(\frac{1}{\tan \left(\theta^{*}\right)}+\frac{1}{g_{-}^{\prime}(0)}\right)+\frac{\gamma_{f}}{\beta \tan \left(\theta^{*}\right)}+\gamma_{f} \frac{\sqrt{1+\left(g_{-}^{\prime}(0)\right)^{2}}}{g_{-}^{\prime}(0)} \geq 0 .
$$

Since $\beta \leq 1$, the previous inequality implies (4.26), which in turn, arguing as in the case of island borders, yields $\theta=\theta^{*}$. This contradicts (4.33).

Consider now the case in which $\theta^{*}=0$, and assume by contradiction that

$$
\theta:=\theta^{-}\left(x_{0}\right)>\theta^{*},
$$

namely $\beta=1$. Then, for $\delta$ small enough, by (4.23), we have $0=\tan \left(\theta^{*}\right)<\delta<-g_{-}^{\prime}(0)$. We define $\psi_{\delta}$ as in the case of island borders, by

$$
\psi_{\delta}(s)= \begin{cases}-\left(\frac{g_{-}^{\prime}(0)+\varepsilon_{\delta}}{\delta}\right) s+\frac{\varepsilon_{\delta}}{g_{-}^{\prime}(0)}+1 & \text { for } \frac{\delta}{g_{-}^{\prime}(0)}<s \leq 0 \\ \frac{-\varepsilon_{\delta}}{\delta} s+\frac{\varepsilon_{\delta}}{g_{-}^{\prime}(0)}+1 & \text { for } 0<s<\delta\left(\frac{1}{\varepsilon_{\delta}}+\frac{1}{g_{-}^{\prime}(0)}\right), \\ 0 & \text { otherwise. }\end{cases}
$$

where $\varepsilon_{\delta}<<\delta$ is such that $\delta\left(\frac{1}{\varepsilon_{\delta}}+\frac{1}{g_{-}^{\prime}(0)}\right)<1$. Analogous computations to the case $\theta^{*}>0$, as well as the fact that $\beta=1$, yield the inequality

$$
-\gamma_{f}\left(\frac{1}{\tan \left(\theta^{*}\right)}+\frac{1}{g_{-}^{\prime}(0)}\right)+\gamma_{f} \frac{\sqrt{1+\varepsilon_{\delta}^{2}}}{\varepsilon_{\delta}}+\gamma_{f} \frac{\sqrt{1+\left(g_{-}^{\prime}(0)\right)^{2}}}{g_{-}^{\prime}(0)} \geq 0,
$$

which is the same relation that we obtained in (4.30). As in Step 2, in the case of island borders with $\theta^{*}=0$, we deduce that $0>g_{-}^{\prime}(0) \geq-\varepsilon_{\delta}$, and, by the arbitrary smallness of $\varepsilon_{\delta}$, that $g_{-}^{\prime}(0)=0$. This contradicts (4.34) and completes the proof of Assertion 1.

Step 3 (Surface-energy convergence under conditions $\left(c_{2}\right)$ or $\left(c_{3}\right)$ ). We point out that conditions $\left(c_{2}\right)$ or $\left(c_{3}\right)$ correspond to $z_{0}$ being a lower-endpoint of a connected component of $\Gamma_{h}^{j u m p}$. In this step we prove Assertion 3. of the proposition, namely we show that conditions $\left(c_{2}\right)$ and $\left(c_{3}\right)$ are never satisfied except when $\beta=0$. As in the previous step we distinguish the case of island borders, of valleys with no vanishing contact angles, and of valleys with one vanishing contact angle. We only consider condition $\left(c_{3}\right)$. The same arguments work under condition $\left(c_{2}\right)$.

Jumps: Island borders. Here we prove that if $\theta^{*} \neq \frac{\pi}{2}$ then there are no jumps at island borders. Assume by contradiction that there exists $x_{0}=c$ for some $(c, d) \in I_{h}$ such that $\theta^{-}(c)=\pi / 2$, and that $\theta^{*} \neq \frac{\pi}{2}$.

We first consider the case in which $\theta^{*}>0$. We choose $\psi_{\delta}$ such that

$$
\psi_{\delta}(s):= \begin{cases}-\frac{\tan \left(\theta^{*}\right)}{\delta} s & \text { for } 0<s<\frac{\delta}{\tan \left(\theta^{*}\right)}, \\ 0 & \text { otherwise }\end{cases}
$$


We observe that

$$
\mathcal{H}\left(\tilde{\Gamma}_{h+\delta \psi_{\delta}^{n}} \cap\{y=0\}\right)-\mathcal{H}\left(\tilde{\Gamma}_{h} \cap\{y=0\}\right)=-\frac{\delta r_{n}}{\tan \left(\theta^{*}\right)}
$$

Therefore

$$
\begin{aligned}
B_{n} & =\frac{\gamma_{f}}{\delta r_{n}} \int_{x_{0}}^{\frac{\delta r_{n}}{\tan \left(\theta^{*}\right)}+x_{0}} \sqrt{1+\left(h^{\prime}(x)+\delta \psi_{\delta}^{\prime}\left(\frac{x-x_{0}}{r_{n}}\right)\right)^{2}} d x-\frac{\gamma_{f} \beta}{\tan \left(\theta^{*}\right)}-\gamma_{f} \\
& =\frac{\gamma_{f}}{\delta} \int_{0}^{\frac{\delta}{\tan \left(\theta^{*}\right)}} \sqrt{1+\left(h^{\prime}\left(x_{0}+r_{n} s\right)+\delta \psi_{\delta}^{\prime}(s)\right)^{2}} d s-\frac{\gamma_{f} \beta}{\tan \left(\theta^{*}\right)}-\gamma_{f} .
\end{aligned}
$$

By the Dominated Convergence Theorem, we obtain

$$
B_{n} \rightarrow-\frac{\gamma_{f} \beta}{\tan \left(\theta^{*}\right)}+\frac{\gamma_{f}}{\beta \tan \left(\theta^{*}\right)}-\gamma_{f}
$$

By (4.9), (4.16), and Step 1, there holds

$$
-1-\frac{\beta}{\tan \left(\theta^{*}\right)}+\frac{1}{\beta \tan \left(\theta^{*}\right)} \geq 0 .
$$

Thus, in view of (4.23) we conclude that

$$
\sin \left(\theta^{*}\right)=\sqrt{1-\beta^{2}} \geq 1
$$

namely, a contradiction.

Consider now the case in which $\theta^{*}=0$, and choose

$$
\psi_{\delta}(s):=-s
$$

for every $s \in(0,1)$. Then,

$$
\mathcal{H}\left(\tilde{\Gamma}_{h+\delta \psi_{\delta}^{n}} \cap\{y=0\}\right)-\mathcal{H}\left(\tilde{\Gamma}_{h} \cap\{y=0\}\right)=-r_{n},
$$

and, since $\beta=1$,

$$
\begin{aligned}
B_{n} & =\frac{\gamma_{f}}{\delta r_{n}} \int_{x_{0}}^{r_{n}+x_{0}} \sqrt{1+\left(h^{\prime}(x)+\delta \psi_{\delta}^{\prime}\left(\frac{x-x_{0}}{r_{n}}\right)\right)^{2}} d x-\frac{\gamma_{f}}{\delta}-\gamma_{f} \\
& =\frac{\gamma_{f}}{\delta} \int_{0}^{1} \sqrt{1+\left(h^{\prime}\left(x_{0}+r_{n} s\right)+\delta \psi_{\delta}^{\prime}(s)\right)^{2}} d s-\frac{\gamma_{f}}{\delta}-\gamma_{f} .
\end{aligned}
$$

By the Dominated Convergence Theorem, we have

$$
B_{n} \rightarrow-\frac{\gamma_{f}}{\delta}+\gamma_{f} \frac{\sqrt{1+\delta^{2}}}{\delta}-\gamma_{f}
$$

Thus, properties (4.9), (4.16), and Step 1 imply that

$$
-1-\frac{1}{\delta}+\frac{\sqrt{1+\delta^{2}}}{\delta} \geq 0
$$

Since $-1-\frac{1}{\delta}+\frac{\sqrt{1+\delta^{2}}}{\delta}<0$ for every $\delta>0$, we reach also in this case a contradiction. 
Jumps: Valleys with no vanishing contact angles. In this subsection we prove that for every $\theta^{*}$ there are no jumps at valleys with no vanishing contact angles. Consider $x_{0} \in P_{h}$ and such that $\theta^{-}\left(x_{0}\right)=\frac{\pi}{2}$. We want to prove that $g_{+}^{\prime}(0)=0$. Assume by contradiction that $g_{+}^{\prime}(0)>0$. Let

$$
\psi_{\delta}(s):= \begin{cases}0 & \text { for } s>\frac{\delta}{g_{+}^{\prime}(0)} . \\ \frac{\delta-g_{\infty}(s)}{\delta} & \text { for } s \in\left(0, \frac{\delta}{g_{+}^{\prime}(0)}\right],\end{cases}
$$

for every $s \in(0,1)$. By the definition of $\psi_{\delta}$ there holds

$$
B_{n}=\frac{\gamma_{f}}{\delta r_{n}}\left(\mathcal{H}\left(\tilde{\Gamma}_{h+\delta \psi_{\delta}^{n}} \cap\{y>0\}\right)-\mathcal{H}\left(\tilde{\Gamma}_{h} \cap\{y>0\}\right)\right) .
$$

In particular, we obtain

$$
\begin{aligned}
B_{n} & =\frac{\gamma_{f}}{\delta r_{n}} \int_{x_{0}}^{\frac{\delta r_{n}}{g_{+}(0)}+x_{0}}\left(\sqrt{1+\left(h^{\prime}(x)+\delta \psi_{\delta}^{\prime}\left(\frac{x-x_{0}}{r_{n}}\right)\right)^{2}}-\sqrt{1+\left(h^{\prime}(x)\right)^{2}}\right) d x-\gamma_{f} \\
& =\frac{\gamma_{f}}{\delta r_{n}} \int_{x_{0}}^{\frac{\delta r_{n}}{g_{+}^{\prime}(0)}+x_{0}}\left(\sqrt{1+\left(h^{\prime}(x)-g_{+}^{\prime}(0)\right)^{2}}-\sqrt{1+\left(h^{\prime}(x)\right)^{2}}\right) d x-\gamma_{f} \\
& =\frac{\gamma_{f}}{\delta} \int_{0}^{\frac{\delta}{g_{+}^{\prime}(0)}}\left(\sqrt{1+\left(h^{\prime}\left(x_{0}+r_{n} z\right)-g_{+}^{\prime}(0)\right)^{2}}-\sqrt{1+\left(h^{\prime}\left(x_{0}+r_{n} z\right)\right)^{2}}\right) d x-\gamma_{f} .
\end{aligned}
$$

By applying the Dominated Convergence Theorem we conclude that

$$
B_{n} \rightarrow-\gamma_{f} \frac{g_{+}^{\prime}(0)}{1+\sqrt{1+\left(g_{+}^{\prime}(0)\right)^{2}}}-\gamma_{f}
$$

as $n \rightarrow+\infty$. Therefore, properties (4.9), (4.16), and Step 1 yield

$$
g_{+}^{\prime}(0) \leq-1-\sqrt{1+\left(g_{+}^{\prime}(0)\right)^{2}},
$$

which contradicts the non negativity of $g_{+}^{\prime}(0)$.

Jumps: Valleys with one vanishing contact angle. Here we prove that if $\theta^{*} \neq \frac{\pi}{2}$ then there are no jumps at valleys with one vanishing contact angle (and hence, by the previous subsection, at every valley). Assume by contradiction that $\theta^{*} \neq \frac{\pi}{2}$, and that there exists $x_{0} \in P_{h}$ with $\theta^{-}\left(x_{0}\right)=\frac{\pi}{2}$ and $g_{+}^{\prime}(0)=0$.

In the situation in which $\theta^{*}>0$ we argue choosing $\psi_{\delta}$ as in the corresponding situation in Step 3, in the case of island borders. The same computations as in that subsection yield

$$
B_{n} \rightarrow-\frac{\gamma_{f}}{\tan \left(\theta^{*}\right)}+\frac{\gamma_{f}}{\beta \tan \left(\theta^{*}\right)}-\gamma_{f} \geq 0
$$

Since $\beta<1$, this implies

$$
-\frac{\gamma_{f} \beta}{\tan \left(\theta^{*}\right)}+\frac{\gamma_{f}}{\beta \tan \left(\theta^{*}\right)}-\gamma_{f} \geq 0,
$$

which in turn yields to (4.36) and to a contradiction.

The situation in which $\theta^{*}=0$ can be dealt with exactly in the same way as in the corresponding setting in Step 3 for island borders. 
We are now ready to prove Theorem 2.3 .

Proof of Theorem 2.3. We observe that Assertion 3. of Theorem 2.3 coincides with Assertion 3. of Proposition 4.1. In the wetting regime $\beta=1$ also Assertion 1. of Theorem 2.3 follows directly from Assertions 1. and 2. of Proposition 4.1. Furthermore, in the dewetting regime $\beta<1$ from Proposition 4.1 for any $(c, d) \in I_{h}$ and $p \in P_{h}$ the angles $\theta^{-}(p), \theta^{+}(p), \theta^{-}(c)$, and $\theta^{+}(d)$ are smaller or equal to $\theta^{*}$ (and at least one between $\theta^{-}(p)$ and $\theta^{+}(p)$ is zero). It remains therefore to assume that $\beta<1$, and in turn

$$
\theta^{*}>0
$$

and to show that for any $(c, d) \in I_{h}$ the angles $\theta^{-}(c)$ and $\theta^{+}(d)$ are not strictly smaller than $\theta^{*}$, and that $P_{h}=\emptyset$.

To this aim we observe that it is enough to show the following claim: for every $z_{0}=\left(x_{0}, h\left(x_{0}\right)\right) \in Z_{h}$ which is a valley or an island border, there holds

$$
\theta^{-}\left(x_{0}\right) \geq \theta^{*} \text {. }
$$

In fact, we already know that in the dewetting regime any $p \in P_{h}$ has at least a zero contact angle from Proposition 4.1.

To show the claim, we argue by contradiction and we assume that there exists a point $z_{0}=\left(x_{0}, h\left(x_{0}\right)\right) \in Z_{h}$ such that

$$
\theta^{-}\left(x_{0}\right)<\theta^{*} .
$$

The case with $\theta^{+}\left(x_{0}\right)<\theta^{*}$ follows by symmetry. We start by defining a competitor profile function $h_{\varepsilon} \in A P(a, b)$ by

$$
h_{\varepsilon}(x):= \begin{cases}h(x) & \text { if } x \notin\left[x_{0}-\varepsilon, x_{0}\right], \\ -\tan \left(\theta^{*}\right)\left(x-x_{0}+\varepsilon\right)+h\left(x_{0}-\varepsilon\right) & \text { if } x \in\left[x_{0}-\varepsilon, x_{0}-\varepsilon+\ell_{\varepsilon}\right], \\ 0 & \text { if } x \in\left[x_{0}-\varepsilon+\ell_{\varepsilon}, x_{0}\right],\end{cases}
$$

for every $x \in(a, b)$ and $\varepsilon>0$ small enough, where the quantity

$$
\ell_{\varepsilon}:=\frac{h\left(x_{0}-\varepsilon\right)}{\tan \left(\theta^{*}\right)}
$$

is well defined owing to (4.37). We observe that $h \geq h_{\varepsilon}$ and that

$$
\left|\Omega_{h}\right|-\left|\Omega_{h_{\varepsilon}}\right| \leq \int_{x_{0}-\varepsilon}^{x_{0}} h(x) d x=\varepsilon \int_{-1}^{0} h\left(x_{0}-\varepsilon y\right) d y
$$

by the change of variable $x=x_{0}+\varepsilon y$. Furthermore, we notice that the integral on the right-hand side of (4.41) converges to zero by the Lebesgue Dominated Convergence Theorem because $h$ is null and continuous at $x_{0}$. Therefore, $\left(u, h_{\varepsilon}\right) \in X$ is admissible for the penalized minimum problem (3.1) for every $\varepsilon>0$ small enough.

From the minimality of $(u, h)$ and Proposition 3.1 it follows that

$$
\begin{aligned}
\mathcal{F}(u, h) & \leq \mathcal{F}\left(u, h_{\varepsilon}\right)+\lambda_{0}|| \Omega_{h}|-| \Omega_{h_{\varepsilon}} \mid \\
& \leq \mathcal{F}\left(u, h_{\varepsilon}\right)+\lambda_{0} \varepsilon \int_{-1}^{0} h\left(x_{0}-\varepsilon y\right) d y,
\end{aligned}
$$

where in the last inequality we again used (4.41). By (1.2), (2.7), (4.39), and (4.40) we obtain

$$
\mathcal{F}\left(u, h_{\varepsilon}\right)=\int_{\Omega_{h_{\varepsilon}}} W_{0}\left(y, E u(x, y)-E_{0}(y)\right) d x d y+\int_{\tilde{\Gamma}_{h_{\varepsilon}}} \varphi(y) d \mathcal{H}^{1}
$$




$$
\begin{aligned}
& +2 \gamma_{f} \mathcal{H}^{1}\left(\Gamma_{h_{\varepsilon}}^{c u t}\right)+\gamma_{f s}(b-a) \\
\leq & \int_{\Omega_{h}} W_{0}\left(y, E u(x, y)-E_{0}(y)\right) d x d y+\int_{\tilde{\Gamma}_{h}} \varphi(y) d \mathcal{H}^{1} \\
& -\gamma_{f} \int_{x_{0}-\varepsilon}^{x_{0}} \sqrt{1+\left(h^{\prime}(x)\right)^{2}} d x+\gamma_{f} \sqrt{h^{2}\left(x_{0}-\varepsilon\right)+\ell_{\varepsilon}^{2}}+\beta \gamma_{f}\left(\varepsilon-\ell_{\varepsilon}\right) \\
& +2 \gamma_{f} \mathcal{H}^{1}\left(\Gamma_{h}^{c u t}\right)+\gamma_{f s}(b-a) \\
= & \mathcal{F}(u, h)-\gamma_{f} \varepsilon \int_{-1}^{0} \sqrt{1+\left(h_{-}^{\prime}\left(x_{0}+\varepsilon y\right)\right)^{2}} d y \\
& +\gamma_{f} \sqrt{h^{2}\left(x_{0}-\varepsilon\right)+\ell_{\varepsilon}^{2}}+\beta \gamma_{f}\left(\varepsilon-\ell_{\varepsilon}\right) .
\end{aligned}
$$

Inequalities (4.42) and (4.43) yield

$$
\begin{aligned}
0 \leq \frac{\lambda_{0}}{\gamma_{f}} \int_{-1}^{0} h\left(x_{0}-\varepsilon y\right) d y- & \int_{-1}^{0} \sqrt{1+\left(h_{-}^{\prime}\left(x_{0}+\varepsilon y\right)\right)^{2}} d y \\
& +\frac{h\left(x_{0}-\varepsilon\right)}{\varepsilon} \frac{\sqrt{1+\tan ^{2} \theta^{*}}}{\tan \theta^{*}}+\beta\left(1-\frac{\ell_{\varepsilon}}{\varepsilon}\right) .
\end{aligned}
$$

By applying again the Lebesgue Dominated Convergence Theorem together with the observation that both $h$ and $h_{-}^{\prime}$ are left continuous at $x_{0}, h\left(x_{0}\right)=0$, and $h_{-}^{\prime}\left(x_{0}\right)=$ $-\tan \left(\theta^{-}\left(x_{0}\right)\right)$, we obtain that

$$
\begin{aligned}
0 \leq-\sqrt{1+\tan ^{2}\left(\theta^{-}\left(x_{0}\right)\right)}+\tan \left(\theta^{-}\left(x_{0}\right)\right) & \frac{\sqrt{1+\tan ^{2} \theta^{*}}}{\tan \theta^{*}} \\
& +\beta\left(1-\frac{\tan \left(\theta^{-}\left(x_{0}\right)\right)}{\tan \theta^{*}}\right) .
\end{aligned}
$$

If $\tan \left(\theta^{-}\left(x_{0}\right)\right)=0$, inequality (4.45) implies that $\beta \geq 1$, which contradicts the fact that $\beta<1$.

Assume now that $\tan \left(\theta^{-}\left(x_{0}\right)\right) \neq 0$. By dividing (4.45) by $\tan \left(\theta^{-}\left(x_{0}\right)\right)$, we have

$$
0 \leq-\frac{\sqrt{1+\tan ^{2}\left(\theta^{-}\left(x_{0}\right)\right)}}{\tan \left(\theta^{-}\left(x_{0}\right)\right)}+\frac{\sqrt{1+\tan ^{2} \theta^{*}}}{\tan \theta^{*}}+\beta\left(\frac{1}{\tan \left(\theta^{-}\left(x_{0}\right)\right)}-\frac{1}{\tan \theta^{*}}\right)
$$

from which we conclude that

$$
\left(\beta \tan \left(\theta^{-}\left(x_{0}\right)\right)-\sqrt{1-\beta^{2}}\right)^{2} \leq 0,
$$

in the same way as done for passing from (4.25) to (4.28). From (4.46) it follows that

$$
\tan \left(\theta^{-}\left(x_{0}\right)\right)=\frac{\sqrt{1-\beta^{2}}}{\beta}=\tan \theta^{*} .
$$

This contradicts (4.38), and therefore the claim and the theorem follow.

\section{REgUlARITY OF LOCAL MINIMIZERS}

In this section we prove Theorem 2.4 by improving the regularity results already contained in Section 3. In particular the results follow from Proposition 3.3, the decay estimate of Proposition 3.6, from implementing some arguments used for Proposition 4.1, and from proving a second decay estimate which is independent from the specific point on the graph $\Gamma_{h} \backslash\left(\Gamma_{h}^{\text {cut }} \cup \Gamma_{h}^{\text {cusp }}\right)$ (see (5.1)). 
Proof of Theorem 2.4. We begin by observing that Assertions 1. and 2. are direct consequences of Proposition 3.3. In fact, as pointed out in [12, Remark 3.6], the only situation in which case (ii) of Proposition 3.3 arises is when $z_{0}$ is either a cusp point or the lowerend point of a vertical cut. Then, by combining Proposition 3.3 with a compactness argument it follows that the set $\Gamma_{h}^{\text {cusp }} \cup\{(x, h(x)): x \in C(h)\}$ where $C(h)$ is the set defined in (2.3) has finite cardinality.

To obtain Assertion 3. we note that, by employing a similar argument to the one of Step 2 (valleys with one vanishing contact angle) of the Proof of Proposition 4.1 in the case of valleys and for the situation of $z_{0}=\left(x_{0}, h\left(x_{0}\right)\right) \in \Gamma_{h} \backslash\left(\Gamma_{h}^{\text {cut }} \cup \Gamma_{h}^{\text {cusp }}\right)$ with $h\left(x_{0}\right)>0$ (by using Step 1 of Proposition 4.1 for $\mathbb{C}_{f}=\mathbb{C}_{s}$ ) we also prove that $\Gamma_{h}^{\text {reg }} \cap\{y>0\}$ is $C^{1}$ and hence, $\Gamma_{h}^{r e g} \backslash Y_{h} \in C^{1}$. In view of this regularity we can implement the argument used in [12], which is based on the following decay estimate: For every parameter $0<\sigma<1$ there exist a constant $C>0$ and a radius $r_{0}$ such that

$$
\int_{B\left(z_{0}, r\right) \cup \Omega_{h}}|\nabla u|^{2} d x d y \leq C r^{2 \sigma}
$$

for all $z_{0} \in \Gamma_{h} \backslash\left(\Gamma_{h}^{c u t} \cup \Gamma_{h}^{\text {cusp }}\right)$ and $0<r<r_{0}$. In view of (5.1) it is possible to prove as in $[12$, Theorem 3.17] that

$$
\mathcal{H}^{1}\left(\Gamma_{h} \cap B\left(z_{0}, r\right)\right) \leq C r^{2 \sigma_{0}}
$$

for $\sigma_{0} \in(1 / 2,1)$ and $r$ small enough. We note that (5.2) follows by a perturbation argument which we can reproduce also in the dewetting regime. In fact by Theorem 2.3 the set $Z_{h} \backslash Y_{h}$ does not include island borders, and so the profile $h$ is only perturbed in $\{y>0\}$. The conclusion then follows from (5.2) by arguing as in the proof of Theorem 6.1 of [3] (see [3, Proposition 6.4]).

Assertion 4. follows as in [12, Theorem 3.19] by taking special care for the case $\mathbb{C}_{f} \neq \mathbb{C}_{s}$. In this case infact, when showing that $h$ is a classical solution of the Euler Lagrange equation (2.9) in $\Gamma_{h}^{r e g} \backslash Z_{h}$ it is not possible to extend the argument to $\Gamma_{h}^{r e g} \backslash Y_{h}$ arguing by approximation. This difficulty is due to the presence of the transmission problem.

\section{ACKNOWLEDGEMENTS}

The authors thank the Center for Nonlinear Analysis (NSF Grant No. DMS-0635983) and the Erwin Schrödinger Institute (Thematic Program: Nonlinear Flows), where part of this research was carried out. P. Piovano acknowledges support from the Austrian Science Fund (FWF) project P 29681 and the fact that this work has been funded by the Vienna Science and Technology Fund (WWTF), the City of Vienna, and Berndorf Privatstiftung through Project MA16-005. E. Davoli acknowledges the support of the Austrian Science Fund (FWF) project P 27052 and of the SFB project F65 "Taming complexity in partial differential systems". Both authors are thankful to Serge Nicaise, and Anna-Margaret Sändig for useful comments on the topic of transmission problems.

\section{REFERENCES}

[1] BAER E., Minimizers of anisotropic surface tensions under gravity: higher dimensions via symmetrization. Arch. Ration. Mech. Anal. 215 (2015), 531-578.

[2] Bellettini G., Kholmatov S., Minimizing movements for mean curvature flow of droplets with prescribed contact angle. arXiv:1612.04175 [math.AP]. 
[3] Bonnet A., On the regularity of edges in image segmentation. Annales de l'I.H.P. Analyse non linaire 13-4 (1996), 485-528.

[4] Bonnetier E., Chambolle A., Computing the equilibrium configuration of epitaxially strained crystalline films. SIAM J. Appl. Math. 62 (2002), 1093-1121.

[5] Caffarelli L.A., Friedman A., Regularity of the boundary of a capillary drop on an inhomogeneous plane and related variational problems. Rev. Mat. Iberoamericana 1 (1985), 61-84.

[6] Chambolle A., Larsen C.J., $C^{\infty}$-regularity of the free boundary for a two-dimensional optimal compliance problem. Calc. Var. Partial Differ. Equ. 18 (2003), 77-94.

[7] Davoli E., Piovano P., Derivation of a heteroepitaxial thin-film model. Submitted 2018.

[8] De Philippis G., Maggi F., Regularity of free boundaries in anisotropic capillarity problems and the validity of Young's law. Arch. Ration. Mech. Anal. 216 (2015), 473-568.

[9] Dryja M., Sarkis M.V., Widlund O.B., Multilevel Schwartz Methods for Elliptic Problems with Discontinuous Coefficients in Three Dimensions. Numerische Mathematik. 72 (1996), 313-348.

[10] Dupré A.M., Dupré P., Théorie mécanique de la chaleur. Gauthier-Villars, Paris 1869.

[11] Fonseca I., Fusco N., Leoni G., Millot V., Material voids in elastic solids with anisotropic surface energies. J. Math. Pures Appl. 96 (2011) 591-639.

[12] Fonseca I., Fusco N., LeOni G., Morini M., Equilibrium configurations of epitaxially strained crystalline films: existence and regularity results. Arch. Ration. Mech. Anal. 186 (2007), 477-537.

[13] Fonseca I., Fusco N., Leoni G., Morini M., A model for dislocations in epitaxially strained elastic films. Preprint (2016), https://arxiv.org/abs/1605.08432.

[14] Fried E., Gurtin M.E., A unified treatment of evolving interfaces accounting for small deformations and atomic transport with emphasis on grain-boundaries and epitaxy. Adv. Appl. Mech. 40 (2004), 1-177.

[15] Fusco N., Morini M., Equilibrium configurations of epitaxially strained elastic films: second order minimality conditions and qualitative properties of solutions. Arch. Ration. Mech. Anal. 203 (2012), $247-327$.

[16] GaO H., Mass-conserved morphological evolution of hypocycloid cavities: a model of diffusive crack initiation with no associated energy barrier. Proceedings of the Royal Society of London 448 (1995), 465-483.

[17] Gauss C.F., Principia generalia theoriae figurae fluidorum in statu aequilibrii. Springer, Berlin, Heidelberg 1877.

[18] Grinfeld M.A., The Stress Driven Instabilities in Crystals: Mathematical Models and Physical Manifestations. J. Nonlinear Sci. 3 (1993), 35-83.

[19] Grisvard P., Elliptic problems in nonsmooth domains. Society for Industrial and Applied Mathematics (SIAM), Philadelphia (PA) (1985).

[20] KneEs D., Regularitätsaussagen für zweidimensionale elastische Felder in Kompositen. Diploma Thesis. University of Stuttgart, 2001.

[21] KneEs D., Regularity results for transmission problems for the Laplace and Lamé operators on polygonal or polyhedral domains. SFB 404, Bericht 2002/10.

[22] KneEs D., SäNDig A.-M., Regularity of elastic fields in composites. Multifield problems in solid and fluid mechanics, 331-360, Lect. Notes Appl. Comput. Mech., 28, Springer, Berlin 2006.

[23] Kozlov V.A., MAz'Ya V.G., Spectral properties of the operator bundles generated by elliptic boundary value problems in a cone. Func. Anal. Appl. 22 (1988), 114-121.

[24] Laplace P.S., Traité de Mécanique Céleste. Supplément au dixième livre du Traité de Mécanique Céleste, 4 (1805), 1-79.

[25] Nicaise S., Sändig, A-M, General interface problems. I, II.. Math. Methods Appl. Sci. 17 (1994), $395-429,431-450$

[26] Nicaise S., SÄNDig, A.-M., Transmission problems for the Laplace and elasticity operators: regularity and boundary integral formulation. Math. Models Methods Appl. Sci. 9 (1999), 855-898.

[27] Pohl U.W., Epitaxy of Semiconductors: Introduction to Physical Principles. Springer-Verlag Berlin, Heidelberg 2013.

[28] Spaepen, F. Substrate curvature resulting from the capillary forces of a liquid drop. J. Mech. Phys. Solids 44(5) (1996), 675-681.

[29] Spencer B.J., Asymptotic derivation of the glued-wetting-layer model and the contact-angle condition for Stranski-Krastanow islands. Phys. Rev. B 59 (1999), 2011-2017.

[30] Spencer B.J., Asymptotic solutions for the equilibrium crystal shape with small corner energy regularization. Phys. Rev. E 69 (2004), 011603. 
[31] Spencer B.J., Tersoff J., Equilibrium Shapes and Properties of Epitaxially Strained Islands. Phys. Rev. Lett. 79 (1997), 4858.

[32] Srolovitz D.J., Davis S.H., Do stresses modify wetting angles? Acta Mater. 49 (2001), 1005-1007.

[33] TAYlor J.E., Mathematical Models of Triple Junctions. Interface Science 7 (1999), 243-249.

[34] Tekalign W.T., Spencer B.J., Thin-film evolution equation for a strained solid film on a deformable substrate: Numerical steady states. J. Appl. Phys. 102 (2007), 073503.

[35] Young T., An Essay on the Cohesion of Fluids. Phil. Trans. Roy. Soc. 95 (1805), 65-87.

[36] Zisman W.A., Relation of the equilibrium contact angle to liquid and solid constitution. Advances in Chemistry, 43 (1964), 1-51.

(Elisa Davoli) Department of Mathematics, University of Vienna, Oskar-Morgenstern Platz 1, 1090 Vienna (Austria)

E-mail address, E. Davoli: elisa.davoli@univie.ac.at

(Paolo Piovano) Department of Mathematics, University of Vienna, Oskar-Morgenstern Platz 1, 1090 Vienna (Austria)

E-mail address, P. Piovano: paolo.piovano@univie.ac.at 